\title{
Probleme des Stufenwechsels im Lappischen
}

Als K. B. Wiklund i.J. 1921 im "Virittäjä» über den dreistufigen Stufenwechsel schrieb, stellte er fest, dass das Systemgebäude der damals allgemein gutgeheissenen Stufenwechseltheorie in vielen Hinsichten Lücken und schwache Stellen aufwies, ja sogar einem Kartenhaus glich. "Nur ansehen, nicht berühren! sagt man, wenn man die Kartenhäuser ansieht, die artige Kinder sich zusimmenbauen", bemerkt Wiklund ein wenig boshaft. Er selbst möchte dennoch die Festigkeit des Gebäudes erproben, denn "es wird doch nicht zusammenbrechen, wemn ich auch ein bisschen darauf blase». Natürlich ist es nicht zusammengebrochen, aber die lautgeschichtliche Forschung hat doch viele Anregungen erhalten, ja sogar wertvolle Beiträge. Damals waren noch die Zeiten, wo die Gemüter bei Fragen des Stufenwechsels leicht aufwallten. Und das ist auch kein Wunder, denn dieser war der beherrschende Mittelpunkt der finnisch-ugrischen Lautgeschichte geworden. Und man mag über die wissenschaftliche Stichhaltigkeit der Theorie sagen, was man will, durch sie und mit ilrr begann jedenfalls insbesondere die Erforschung der Lautgeschichte der ostseefinnischen Sprachen und des Lappischen, sich zu einer kritischen Wissenschaft zu entwickeln. Die Zeiten haben sich geändert. Heutzutage kann man über den Stufenwechsel Meinungen äussern, welche immer man will, ein Sturm erhebt sich sicherlich nicht. Das Interesse der Forscher wendet sich allmählich von der Lautgeschichte ab, keinesfalls ist sie mehr in einer so dominierenden Stellung wie früher, und der Vokalismus erobert sich ja auch in der Lautgeschichte die Beachtung, die ihm unverdienterweise bisher nicht zuteil geworden war. Es wäre jedoch kein Glück für die Forschung, wenn man die Fragen des Stufenwechsels ganz und gar beiseite liesse und 
dächte, dass das Wichtigste schon gesagt sei. Der Stufenwechsel hat meiner Meinung nach doch immer noch die Schlüsselstellung in der finn.-ugrischen Forschung mit noch grösserem Recht als der Ablaut im Indoeuropäischen.

Wie viele erstrangig wichtige und immer noch auf Lösung harrende Probleme mit den Stufenwechselerscheinungen des Lappischen verbunden sind, zeigt recht gut die gründliche Untersuchung L'alternance consonantique date-t-elle du lapon commun? (Studia Septentrionalia 2), die Knut Bergstand in der 1945 erschienenen Festschrift für Konrad Nielsen veröffentlicht hat. Diese mit ausgezeichnetem Scharfblick geschriebene, ein weites Wissen verratende Abhandlung ist, wie mir scheint, als ein sclbständiger Beitrag zur Diskussion gemeint, um die mannigfaltigen Zweifel zum Ausdruck zu bringen, die im jungen Forscher aufstiegen, als er in die vielseitigen lautgeschichtlichen Probleme des Lappischen eindrang, mehr als eine irgendwie endgültige Stellungnahme. Die Diskussion fliesst jedoch auf unserem Wissenschaftsgebiet so träge dahin, dass man ein neues Aufgreifen der Fragen gewöhnlich von einer ganz neuen Generation oder wenigstens erst nach Jahrzehnten erwarten kann. In der im Folgenden vorgelegten Untersuchung sollen die Gedanken zur Sprache kommen, die mir während einer längeren Zeit immer wieder in den Sinn kamen, als ich die stimulierenden Ausführungen Bergslands durchdachte. Zwei miteinander zusammenhängende Fragegruppen stehen in erster Linie im Vordergrund meiner Darstellung. Zunächst als allerwichtigste die Frage, ob der Stufenwechsel in den lappischen Dialekten ein Erbe aus dem Urlappischen ist, und zweitens, welcherart dieser Stufenwechsel ursprünglich gewesen ist.

Ist der stufenwechsel urlappisch?

In einer Beziehung bedeutet die Untersuchung Bergslands einen beachtenswerten neuen methodischen Beitrag. Er unterscheidet nämlich von dem rein phonetischen Stufenwechsel den phonematischen, den er für den eigentlichen Stu- 
fenwechsel hält. Das ist meiner Meinung narh ein besonders wichtiger Umstand, denn viele grundsätzliche Fragen müssen eben von diesem Standpunkt her geprüft werden. In der bisherigen Theorie hat man keinen derartigen Unterschied gemacht. Der Stufenwechsel wurde gleichzeitig sowohl als ein phonetischer wie auch phonematischer dargestellt. Wenn man sagt, dass die Geminataklusile mit Geminaten, deren erster Teil kurz ist, gewechselt haben (z.B. katto : kalton), handelt es sich natürlich um den Wechsel von Varianten oder Allophonen desselben Phonems; eine Geminata mit kurzem Anfangsteil trifft man niemals in derselben lautlichen Umgebung an wie eine lange Geminata und umgekehrt. Ihre Distribution ist also komplementär, sie gehören zu demselben Phonem, so dass man sie in der phonematischen Transkription mit demselben Zeichen bezeichnen kann. Wenn man dagegen annimmt, dass das $t$ der starken Stufe mit dem $\delta$ der schwachen Stufe gewechselt habe, handelt es sich nicht mehr um den Wechsel zweier Varianten, sondern zweier Phoneme, weil es Wörter gibt, bei denen auch in der starken Stufe der Dentalspirant steht.

Die Unterscheidung des phonetischen und des phonematischen Stufenwechsels ist besonders wichtig, wenn man den Schwund des Stufenwechsels untersucht. Es ist klar, dass der phonetische Stufenwechsel schwindet, ohne Spuren zu hinterlassen; handelt es sich doch nur um den Ausgleich verschiedener Varianten desselben Phonems, der unter dem Einfluss einer' andersartigen Aussprache leicht stattfinden kann. Dagegen kann der phonematische Stufenwechsel kaum verschwinden, ohne dass irgendwelche Spuren blieben. Ich bin ganz derselben Meinung wie Bergsland darüber, dass der phonematische Stufenwechsel eine junge Erscheinung ist. Dagegen halte ich den phonetischen Stufenwechsel, wie ich in Virittäjä 1951 dargelegt habe, für urfinnisch-lappisch.

Den Sprachgeschichtler interessiert natürlich der phonetische Stufenwechsel ebensosehr wie der phonematische, gelegentlich vielleicht sogar noch mehr, weil man durch ihn gleichsam zu den ersten Quellen der Erscheinung vordringt. Ausserdem ist die Grenze nicht immer leicht zu ziehen. Man denke sich z.B., dass der Wechsol katto : katton sich zu katto : katon 
wandelt dadureh, diass dir Geminata mit kurzen Anfangstril sich zu einem einfachen Klusil entwickelt, wie es im Finnischen ja geschehen ist. Nun handelt es sich natürlich um den Wechsel zweicr verschiedener Phoneme, denn neben dem Morphem katto rribt es auch kato, das einen ursprünglichen einfachen Klusil repräsentiert. Aber wenn man die Geminata niemals vor einer geschlossenen Silbe antrifft, sondern an deren Stelle immer einen einfachen Klusil, kann man diesen einfachen Klusil in einem bestimmten Sinne auch weiterhin für eine Variante der (reminata halten. Xichts würde uns hindern, fortgesetzt phonematisch / katto: katton! zu schreiben, wenn ein klares Gesetz zeigt. dass $t$ vor einer geschlossenen Silbe als einfaches $t$ auszusprechen ist. Ebenso könnte man schreiben: lato : katon, obgleich es sich phonetisch um den Wechsel [kato : kadon] handelt, oder warum nicht auch lato : katon, und kado: kadon.. Wenn wir auch auf ein sogen. "overlapping" hinweisen und sagen könnten, dass dasselbe Allophon zu zwei verschiedenen Phonemen gehören kann, ändert das doch nicht die Tatsache, dass die Grenze zwischen dem phonetischen und dem phonematischen Stufenwechsel nicht immer schroff ist. Wichtig ist, wie weiter unten noch eigens dargelegt wird, dir Aufmerksamkeit darauf zu richten, dass der phonetisch gleiche Laut im System der Sprache verschiedene Funktionen haben kann und dass z.B. $t$ sich in einigen Formengruppen mit der Geminata $t t$ und in einigen anderen wieder mit dem stimmhaften $d$ assoziiert.

Die Lappologen zeigen eine seltene Einmütigkeit darin, dass der Stufenwechsel tatsächlich ein urlappisches Erbe ist. Das ist auch gut zu verstehen, da er im Prinzip ganz gleichartig in allen lappischen Dialekten - mit Ausnahme des Südlappischen - zu finden ist. Der lappische Stufenwechsel ist in seinen wesentlichen Zügen ganz die gleiche Erscheinung wie im Ostseefinnischen, wo der Stufenwechsel ganz allgemein überall verbreitet ist. Nur das Wepsische und Livische bilden eine Ausnahme. Diese sprachen, die den Stufenwechscl nicht aufweisen, waren in erster Linie der Anlass zu den polemischen Zweifelsäusserungen, die Lauri Ketтuxex in verschiedenen Zusammenhängen gegen die Annahme, der Stufenwechsel 
sei urfinnisch, gerichtet hat. Ebenso wie das Wepsische und Livische Kettunen an dem Stufenwechsel des Urfinnischen zweifeln liessen, hat nun also das Südlappische Bergsland zu den gleichen Schlussfolgerungen bezüglich des Urlappischen geführt. Es liegt in der Natur der Sache, dass auch bei der Beweisführung in einigen Punkten dieselben Linien befolgt werden.

Der lappische Stufenwechsel hat in der Hinsicht eine Besonderheit, dass er den Stammkonsonantismus in seiner Ganzheit umfasst (z.B. IpN. guolle : guole $\sim$ fi. lala : kalan) und nicht bloss die Klusile. Dieser besondere Zug des lappischen Stufenwechsels, den man also im Ostseefinnischen nicht kennt, ist all den lappischen Dialekten gemeinsam, die den Stufenwechsel haben. Deswegen darf man es wohl als sicher betrachten, dass der Stufenwechsel im Lappischen jedenfalls in einer 7eit entstanden ist, wo wenigstens die heutigen Dialekte mit Stufenwechsel in engerer Berührung miteinander gestanden haben und praktisch gesehen ein gemeinsames Sprachgebiet bildeten. Deshalb ist es auch keineswegs denkbar, dass ein starker Einfluss des Finnischen von verschiedenen Seiten her im Lappischen den Stufenwechsel hervorgebracht habe. Der Stufenwechsel des Lappischen ist im ganzen genommen eine lappische Erscheinung, seine Entwicklung und sein Vorkommen muss vor allem im Lichte der eigenen Vorbedingungen des Lappischen selbst verstanden werden. Wenn der Stufenwechsel des Lappischen und des Ostseefinnischen aus einer gemeinsamen Quelle stammt, wie der Schreiber dieser Zeilen voraussetzt, bedeutet dies, dass man diese Quelle in einer recht fernen Vergangenheit suchen muss, zum mindesten in der finn.lappischen Ursprache. Damit ist natürlich ohne weiteres klar, dass das Fehlen des Stufenwechsels im Wepsischen, Iivischen und Südlappischen deutlich späten Ursprungs ist. Wir können jedoch nicht die vermutete gemeinsame finnisch-lappische Herkunft des Stufenwechsels zum Ausgangspunkt unserer Untersuchung machen. Wir richten unsere Aufmerksamkeit jetzt nur auf die Verhältnisse im Lappischen und versuchen, in dieser Sprache selbst die Kriterien zu finden, die in unswe? Frage Licht bringen können. 
Zunächst können wir fragen, ob es z.B. auf Grund aussersprachlicher Fakta auch nur wahrscheinlich oder überhaupt mörrlich ist, lass das Südlappische sich von der übrigen Sprachgemeinschaft so früh getrennt hätte, dass der Stufenwechsol sich darin nicht hättr festsetzen können. An sich wäre das keineswegs unmöglich. denn die Auflösung des Lappischen in zahlreiche Dialekte ist offensichtlich sehr früh erfolgt, wie sich ja auch die lappischen Siedlungen weit über das ganze nördliche Fennoskandien hin ausgebreitet laben; siehe meinen Artikel in der Festschrift für Hermann Hirt II S. 97 ff. Was speziell die sürllappen betrifft. so wissen die geschichtlichen Quellen ron ihrem verhältnismässig späten Erscheinen in den südlichen (regronden schwedens und Norwegens, in denen man sie heutzutage vorfindet. zu berichten. Es ist in den 60-er Jahren des 16. Ths. cin Wandorn der Iappen nach süden in den Fjellgrerenden von Jämtland und Härjedalen festzustellen; siphe K. B. Wrkusw) Huru länge har det funnits lappar i Jämtland och Härjedalen, Festskr. till E. Festin, 1928. In den entsprechenden rebieten auf der norwegischen seite gelangt man auch nicht weiter als bis zum Ende des 16. Jhs.; siehe Kr. Nissen De eldste historiske vidnesbyrd om lapper i NordTrondelag, Årb. for N.-Tr. historielag 1929. Auf Grund einiger ()rtsnamen hat K.B. Wiklund vermutet, die Lappen seien in der urnordischen Zeit auf ihren Wanderungen nach Süden his zum nördlichen Teil des strömtales vorgedrungen und hätten das nördliche Jämtland vor $500 \mathrm{n}$. Chr. erreicht. Aber die urnordische Zeit lässt sich doch als geschichtlicher Zeitabschnitt nur sehr unscharf umgrenzen. Gutory frJessixg hat wegen des Fundes einiger Zaubertrommelschlägel es für möglich gehalten, dass Lappen schon im 15. Jh. in Övre Rendal in Norwegen entweder fest oder vorübergehend gewohnt haben; siehe Studia Septentrionalia 2 S. 99 ff. Im Ångermanland zahlten die Lappen im 16 . Jh. Steuern und im 17. Jh. sind sie schon in Idre, dem heut südlichsten Ort in Dalarne.

Keincrlei geschichtliche oder prähistorische Merkmale berechtigen uns zu dem Schluss, dass die Südlappen sich schon recht früh von ihren nördlichen Stammesbrüdern getrennt hätten und ron ihnen gänzlich isoliert worden wären. Aber 
andrerseits ist klar, dass sie wegen ihrer periphären Lage und wegen der dünnen Besiedelung und grenzenlosen Weite der geographischen Gebiete Jahrhunderte hindurch in ihrer Entwicklung in vieler Hinsicht ihre eigenen Wege gegangen und ausserhalb des Einflussradius der Lappenzentren geblieben sind. So ist es auch von vornherein klar, dass man in ihrem Dialekt einerseits Altes, was anderswo geschwunden ist, andrerseits Neuerungen, die anderswo unbekannt sind, findet.

Aber all dies trifft, vielleicht in noch grösserem Masse, auch für die Ostlappen zu. K. B. WikLuxd hat, besonders mit Berufung auf alte skandinavische Lehnwörter, zu zeigen versucht, dass die Verbindungen der Ostlappen mit dem Westen schon ungefähr $700 \mathrm{n}$. Chr. abgebrochen worden seien. Die Sache ist jedoch nicht ganz so einfach; siehe T. I. ITKovex JSFOu 60,5 S. $18 \mathrm{ff}$. Jedenfalls ist klar, dass der Anschluss an einen ganz anderen politischen und kulturellen Bereich und an eine andere Kirche geeignet war, die Grenzen nach Westen hin abzusperren.

Für die Beurteilung des Alters des Stufenwechsels gibt es also keine derartigen aussersprachlichen Fakta, aus denen sich etwas Bedeutendes herausholen liesse. Die eigentlichen Entscheidungen müssen mit Hilfe der Tatsachen getroffen werden, die der Vergleich der Lauterscheinungen der Dialekte sichtbar werden lässt, und auf Grund der Folgerungen, die man aus den Vergleichen ziehen kann. In der Hauptsache bewegt man sich auch in diesen Fragen im Bereich von Wahrscheinlichkeiten, etwas unbedingt Bindendes oder ganz Eindeutiges ist schwer, wenn nicht geradezu unmöglich, zu finden. Vor allem muss man meiner Meinung nach davon ausgehen, dass der Stufenwechsel zu einer Zeit entstanden ist, wo die ostlappischen Dialekte noch in naher Berührung mit dem Finnmarklappischen standen und dieses seinerseits mit den schwedischlappischen Dialekten bis zum Umelappischen hin, in dem der Stufenwechsel noch zu finden ist. Es handelt sich also darum, ob es auf Grund sprachlicher Tatsachen wahrscheinlich ist, dass das Südlappische (also die in Jämtland und Härjedalen gesprochenen Dialekte) sich so früh aus der Gemeinschaft mit den übrigen Dialekten gelöst habe, diss die Wechselbeziehungen der letztgenannten auch noch nach dieser Trennung verhältnismässig 
stark fortgedauert haben. Etwas derartiges muss man voraussetzen, wenn man meint, dass der Stufenwechsel nach der urlappischen Epoche in irgendeinem Mittelpunkt seinen Anfang genommen und sich sodann von dort in die Nachbardialekte verbreitet habe. Die Verbindungen nach dem äussersten Süden wären damn, wenn nicht direkt abgebrochen, so doch wenigstens in dem Masse schwächer geworden, dass eine solche Neuerung wie der Stufenwechsel nicht mehr hätte Fuss fassen können. Wenn wir annehmen. dass seine Verbreitung von einem Dialekt zum anderen nach Art der Lehnbezielnungen vor sich rringr, setzt dies natürlich eine sehr enge gegenseitige Beeinflussung voraus. Wenn ich zu dem schluss gekommen bin, dass kein Anlass besteht zu denken, die ostlappischen Dialekte seien mit den zentralen Dialekten, bei denen der Stufenwechsel herrschte, länger in Berührung geblieben als die südlappischen, und dass kein begründeter Anlass bestehe anzunelımen, gerade diese südlichen Dialekte hätten sich tatsächlich als allererste von der ursprünglichen Sprachgemeinschaft getrennt, so beruht das zum Teil darauf, dass ich die Grenze, die das Umelappische rom Südlappischen trennt, ganz und gar nicht als fest betrachten kann. Es ist nicht möglich zu meinen, dass alle die zahlreichen z.T. besonders charakteristischen Züge, die das Südlappische mit den diron nördlicher befindlichen Dialekten verbinden, die aber auf ostlappischem Gebiet nicht mehr zu finden sind, durch spätere Einwanderungen mitgebracht worden seien.

Obgleich man nirgendswo aluf dem ganzen lappischen Sprachrebiet von unbedingt schroffen Dialektgrenzen sprechen kann, sondern überall Zwischenstufen und Übergangserscheinungen festzustellen sind, ist es doch sehr wahrscheinlich, dass die älteste Dialektgrenze, wie man angenommen hat. sich gerade zwischen den ostlappischen und den westlappischen Dialekten gebildet hat. Man muss sich daran erinnern, dass die sogenannten seelappischen Dialekte, die vom Varangerfjord bis weit nach Westen in der Gegend von Tromsö gesprochen wurden, offensichtlich ostlappisch gewesen sind. Von diesen Dialekten sind sicherlich viele bleibende Spuren im Finnmarklappischen geblieben, und diese sind ihrerseits geeignet, die Grenze zwi- 
schen den ost- und den finnmarklappischen Dialekten weniger schroff zu machen, als sie vielleicht sonst wäre. Wenn man klar beweisen könnte, dass die schärfste Dialektgrenze zwischen den ost- und den westlappischen Dialekten hindurchgeht, und dass die erste entscheidende Teilung des Sprachgebietes sich längs dieser Grenze vollzog, wäre das ein ausgezeichnetes, schwerwiegendes Argument für den urlappischen Ursprung des Stufenwechsels.

Von den Lauterscheinungen, die die südlappischen Dialekte mit den anderen westlappischen Dialekten fest verbinden im Gegensatz zu den ostlappischen Dialekten, möchte ich zunächst den bekannten Enterschied in der Behandlung des finn.ugrischen ${ }^{*} s k$ und ${ }^{*} s t$ erwähnen. Im Ostlappischen ist bis zum Inarilappischen und den Mundarten von Sompio $\check{s}$ die Entsprechung von ${ }^{*} \dot{s}$, aber im Finnmarklappischen bis zu den südlichsten schwedischlappischen Dialekten ganz regelmässiır $i$, z.B. Ip $N$ vei ke, lpK viešlie, finn. vaski, mordw. ushíe usw. Weitere Beispiele siehe bei Äıм̈̈ Astevaihtelututkielmia S. $128 \mathrm{ff}$. Auch die seelappischen Dialekte scheinen früher diesen Lautstind vertreten zu haben, wie man aus der Mitteilung K. LeEms, dass im alten seelappischen Dialekt von Porsanger ${ }^{*} s k$ und ${ }^{*} s t$ durch $s k$ und $s t$ vertreten werden, schliessen kann (En Lappisk (trammatica), siehe J. Qvigstad Die lappischen Dialekte S. 11. ${ }^{1}$ Diesem Lautphänomen und seinem besonders klar begrenzten Vorkommen ist bei der Klarstellung der Bezichungen zwischen den lappischen Dialekten recht grosses Gewicht beizulegen. Es verbindet die südlappischen Dialekte eng mit den westlappischen im Gegensatz zu den ostlappischen. Wenn wir uns auf den Standpunkt von E. N. SETÄLä stellen und erklären, dass das $i$ ' und $i t$ der westlichen Dialekte aus der schwachen Stufe ron ${ }^{*} z k$ und ${ }^{*} z i t$ und das $s k$ und $s ̌ t$ der

1 Es scheint wahrscheinlich, dass das von NIELSEN erwähnte $b a \grave{a} \breve{s}^{\prime} k \hat{a}$, das in den Mundarten von Polmak, Karasjok und Kautokeino in bildlicher Bedeutung 'dirt (fig.), good-for-nothing, 'skitt' i fig. bet. (oftest brukt i bebreidende tiltale til eller i omtale av barn el. husdyr)' neben bâika vorkommt, und das Verb bas'lket neben der zu erwartenden Form bâiket aus seelappischen Dialekten an der Küste übernommen worden sind. 
östlichen Dialekte hingegen aus einer Verallgemeinerung der starken Stufe entstanden sind, dann würde das ohne weiteres bedeuten, dass das Südlappische sich auf einen früheren Stand des Stufenwechsels zurückführen lässt. Man kann sich jedoch kaum auf den Stufenwechsel berufen, wenn man die verschiedene Vertretung der Dialekte klarstellen will. Offensichtlich handelt es sich um einen klaren Lautwandel: Im Westen hat sich $i^{i} k, i^{\prime} t$ in allen Stellungen entwickelt, und im Osten $̌ k$, št. Es ist nicht denkbar, dass die südlappischen Dialekte zul der Zeit, als die in Rede stehende recht bedeutsame Lautveränderung durchgeführt wurde, vom Einfluss der Stufenwechselerscheinung unberührt geblieben wären, denn es ist ganz klar, dass der Stufenwechsel älter ist als der genannte Lautwandel. Wenn es nicht so wäre, müsste man den Stufenwechsel der ostlappischen Dialekte für eine selbständige Erscheinung halten und das ist m.E. ganz unmöglich.

Es ist noch eine andere Lauterscheinung zu nennen, die ihrer Natur nach dieselbe ist, wie die eben dargestellte. Ich meine den in den westlappischen Dialekten vorkommenden klusilen Vorschlag, der vor inlautende Nasale getreten ist, z.B. $1 \mathrm{pN}$ ibmel 'Gott', fi. jumala usw. Der im Inarilappischen vorkommende kurze klusile Vorschlag ist vermutlich eine Ausstrahlung des Finnmarklappischen und stellt eine der frühesten Entwicklungsphasen dar; weiter im Osten ist der Vorschlagslaut ganz unbekannt, während er eine normale Erscheinung in allen südlicheren Dialekten ist.

Diese sind jedoch nicht die einzigen Isoglossen, die die westlichen lappischen Dialekte vom Südlappischen bis zum Finnmarklappischen in einer ganz wesentlichen Weise verbinden, wenn sie auch wohl am meisten in die Augen fallen. Es sei noch der Wechsel $o \sim u$ in der zweiten Silbe in den westlappischen Dialekten erwähnt, wobei das $u$ unter dem Einfluss eines engen Vokals in der dritten Silbe entstanden ist, wie K. B. Wiklund gezeigt hat; siehe FUF $241 \mathrm{ff}$. Erkki IтkoNEx hat festgestellt - siehe Der ostlappische Vokalismus vom qualitativen Standpunkt aus S. 113 - dass die ostlappischen Dialekte einen solchen Wechsel nicht kennen und auch sicherlich nicht gekannt haben, vgl. jedoch Bergstand Roros-lappisk grammatikk S. VIII Fussnote 4. 
Ein gemeinsamer und charalteristischer Zug der ostlappischen Dialekte ist der Schwund der Verbindung * $n_{5}^{2}$ vor einer yeschlossenen Silbe weiter im Wort, z.B.lpI a $\bar{l} \bar{a} \bar{a}$ GASg. von

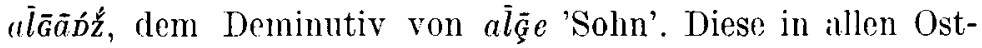
dialekten bekannte schwunderscheinung ist den westlichen Dialekten fremod. K. B. Wikuvxi hat allerdings finmmarklapp. olmuš, GASg. ol'bmu für einen L̇berrest früherer dem Ostlappischen entsprechender Verhältnisse gehalten (vol. lp I olìño'ź, ( deutende Vertretung findet man bis zur Mundart von Gällivare, aber nicht südlicher. In seiner Untersuchung bringt Bergsbaxo jedoch aus dem Finmmarklappischen viele früher ohne Beachtung und Erklärungr grebliebene Fälle vor, in denen es sich nach ihm um den Schwund des alten $* \dot{n} \frac{3}{3}$ handelt, wie

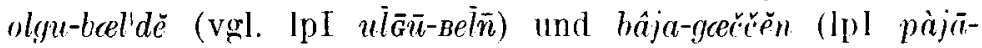
gettsin) usw. Magres sich nun mit dem Schwund des vermuteten * $\dot{n} \breve{3}$ im Finnmarklappischen verhalten haben wie immer es wolle, olmuš kann keinesfalls mit den Ietzterwähnten Fällen zusammengehören, weil hier die starke stufe der schwachen dieser anderen gegenübersteht. olmuš $\sim$ ol'bmu kann grut aus der Mischung zweier Paradigmen entstanden sein, die oblirquen Kasus weisen nämlich am ehesten auf die Form eines i-Stammes. Vgl. das nahestehende almai, olmai, das weithin auch in den Ostdialekten $j$-stämmig ist.

Es ist ausserordentlich wichtig, dass Bergsland die merkwürdigen olgu-, lâja-, jievta- und andere Fälle in die Diskussion gebracht hat, denn sie bedürfen offensichtlich einer Erklärung. Weiter im Westen treffen wir die Formen mit š ganz erwartungsgemäss in allen Stellungen, z.B. lp $\perp^{*}$ (Lenv.) olgušhal'dě, lpL vuoläsj pielēn, lpP vuolaš piellai. Nur in den ostlappischen Dialekten finden sich mit der Vertretung im Finnmarklappischen vergleichbare Formen. Offenbar gehören die Fälle mit $s$ und die mit Schwund zusammen. Jene müssen als Nominativ und diese als Genetiv gedeutet werden.

Das Attribut kongruiert regelmässig nicht im Lappischen, die Pronomina, Zahlwörter und das Adjektiv buorrĕ ausgenommen. Erwartungsqemäss findet man denn auch den Nominativ, von den westlichsten Mundarten des Finnmarklappischen angefangen bis zum Süden. Bergsland erklärt, dass die gene- 
tivische Forn des Attributs dem Einfluss der Demonstrativpronomina zuzuschreiben ist, vol. IpN dām bal'dè, dâm räjest. Diese Erklärungsmöglichkeit begegnet aber grossen Schwierigkeiten. Es ist deutlich zu sehen, dass der Ausgleich des vermuteten Wechsels mit ${ }^{*} n \tilde{5}$, falls es überhaupt einen solchen gegeben hat, im Westen sehr früh stattgefunden hat. In verschiedenen Ableitungen, in denen dieses Element vorkommt, sind keine Spuren davon zu sehen, ebenso hat der Potentialis in seiner Gesamtheit den stand analoger Verallgemeinerung angenommen. Wenn das Finnmarklappische tatsächlich auf einen Stand zurückzuführen ist, wo es einen solchen Wechsel gab, dann konnten zu einer so späten Zcit, in der der Einfluss des Demonstrativpronomens in Frage kommen konnte, von der schwachen, d.h. der Schwundstufe nur wenige erstarte und zum Paradiorma nicht gehïrende spuren vorhanden sein. In Verbindung mit den Inessir und Elativ ist der Genetiv-Akkusativ des attributiven Demonstrativpronomens sichtlich eine verhältnismässig junge Erscheinung. Es wäre wahrhaftig sehr merkwürdig, wenn z.B. in Fällen wie ruolaš, bâjaš usw. der rernutungsgemässe alte lautgesetzliche Genetir mit seiner Schwundvertretung sich so lange hätte erhalten können, dass der vermutete Einfluss des Pronomens ihn überhaupt in die attributive Stellung hätte bringen künnen.

Meines Erachtens kann man die Sache am natürlichsten so erklären, dass es sich nicht um eine Analogie sondern um ein wirkliches altes Crenetivattribut handelt. bâja- ınd ruola- sind also alte Genetivformen von substantivisch gebrauchten Adjektiven bâjaš und ruolaš usw. (Vgl. z.B. fi. pohjoisen puolella pohjoispuolella, pohjoisen puolelle $~$ pohjoispuolelle usw.). Dass es sich tatsächlich so verhält, kann man leicht aus solchen pluralischen Formen wie dāla ol'bmuk 'die heutigen Leute' sehen. Auch hier haben wir eine Genetivform, aber mit einem Demonstrativpronomen heisst es dâk ol'bmuk, also eine regelmässige Kongruenzerscheinung.

Einem möglichen Schwundfall von $*_{n} \tilde{j}$ auf südlappischem Gebiet muss man berechtigterweise nähere Aufmerksamkeit zuwenden. Aus dem Dialekt von Rüros hat Bergsland interessante Formen des Perfektpartizips vorgebracht; siehe Roros- 
lappisk grammatikk \$. 160. Die Endung de's Perfektpartizips gleichsilbiger Vurben ist -ma, die der ungleichsilbigen hingegen -mma, anders als allgemein im südlappischen. Diese Endung - mma ist nach Bergsland nur so zu erklären, dass *n nj an ihr geschwunden ist. Er hält es, freilich zwiifelnd, für möglich. dass die ursprüngliche Form *-mañ sich tatsächlich um einen Schwund nach Art der Ostdialekte handeln würde. ERkKI ITKoxex heisst den Gedanken gut, dass beim Perfektpartizip ungleichsilbiger Verben auch im Südlappischen von einer Form mit dem Suffix *n் ${ }_{3}$ wie im Ostlappischen auszugehen ist, abcr er hält den schwund des ${ }^{*} n .5$ für eine ganz regelwidrige Erscheinung. Er sagt MSFOu 98 S. 275: "Das südlappische -mma beruht ohne Zweifel auf einer" unregelmässigen Verstümmelung. Der Schwund des -ńnźzeElements ist aus zwei Gründen verständlich: erstens war -níźz nicht als Ausdruck der Funktion notwendig, weil das vor ihm stehende -ma- schon allein das Part. Perf. ausdrückte; zweitens hat wahrscheinlich die Analogie der ursprünglich auf einen Vokal endenden Form des Part. Perf. der gleichsilbigen Stämme ihre Wirkung ausgeübt.» Wenn man tatsächlich davon ausgehen muss, dass das Südlappische, genauer gesagt, der Dialekt ron Röros die ursprünglichen Verhältnisse bewahrt habe und sein Perfekt auf die Form mit ${ }^{*} \dot{n} \breve{\jmath}$ zurüchgehe, dann ist kaum eine andere Erklärung zu finden als die von Itkonen gegebene. Meinerseits halte ich es jedoch ganz und gar nicht für klar, dass das Perfektpartizip der ungleichsilbigen Verben im Südlappischen wirklich zu demselben Typ zu rechnen ist wie die entsprechenden Formen der ostlappischen Dialekte.

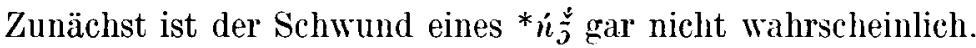
Zweitens scheint sich für die Formen des Perfektpartizips eine viel natürlichere Erklärung ganz aus der Nähe anzubieten. Im. Dialekt von Röros haben, wie Bergsland dargetan hat, die Endungen des Präsens allgemein die Endungen des Imperfekts beseitigt und die 3. P. Sg. des Imperfekts, die der rrine Tempusstamn ohne irgendwelche Personalendungen war,

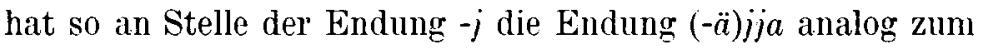
Präsens erhalten, z.B. Essand vardašij, aber Röros vardašäjja 'se etter, betrakte, undersøke', Essand bötajij, Röros bätzjäjja 
'komme'. Auch der Dialekt von Härjedalen, der in grossen ganzen der gleiche ist wie der von Röros, kennt das analogisch

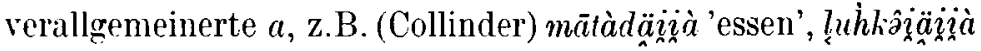
'lesen'. Wie Bergsland erklärt hat, ist dieses a deutlich eine Analogie zum Präsens, siehe Roros-lappisk grammatikk S. 159. Es ist meiner Meinung nach ganz begründet anzunehmen, dass dasselbe $a$ auch in das Perfektpartizip eingedrungen ist. Wie bekannt, erscheint das Perfektpartizip im Südlappischen als Prädikit ohne das Hilfsverb "sein», z.B. (Bergsland) bahliamdamma 'har varmet opp'. Als im Imperfekt das a in der 3 . P. aufzutreten begann, war die analoge Übertragung ganz natürlich, zumal der Gebrauch des Perfektpartizips als Prädikat gerade dann ganz gewöhnlich ist, wenn es sich um die 3. P. Sg. handelt. Es ist zu vermuten, dass das Perfektpartizip gerade dann, wenn es sich um die 3. P. Sg. handelte, schon im Urlappischen als Prädikat ohne Kopula gebraucht worden ist; siehe ERkki Itkonen MSFOu 98 S. 302. Da meiner Meinung nach der Dialekt von Röros demnach in der Bildung des Perfektpartizips der ungleichsilbigen Verben mit dem Ostlappischen nicht auf eine Linie gebracht werden kann, dürfte es nicht begründet sein, das Perfektpartizip vom Typ -mañ nahme der Formen des Verlos la- für urlappisch zu halten. Es ist sogar gut denkbar, dass das Partizip des cinsilbigen Verbs "sein" wegen seines häufigen Gebrauchs Vorbild für die ostlappischen dreisilbigen Verben geworden ist. Auch hier hätten wir also einen Zug, der neben verschiedenen anderen Erscheinungen die ostlappischen Dialekte als Gegenstück zıl den westlichen Dialekten zusammenschliesst. Letzten Endes können die bâja-, ruola- Fälle im Finnmarklappischen sehr gut Entlehnungen aus den seelappischen Dialekten sein.

Im Stufenwechselsystem der ostlappischen Dialekte gibt es zahlreiche Phänomene, die nur dieser Gruppe eigen sind; siehe Erki Itronex FUF 27 S. 137 ff. Besonders möchte ich den Stufenwechsel der Sibilanten erwähnen, der in der schwachen Stufe einen stimmhaften, oder halbstimmhaften Spiranten aufweist, z.B. lpI pōšsą $:$ pōząm (vgl. lpN bâssât:bâsâm). Ohne in diesem Zusammenhang zu der Frage, ob der Typ des Wechsels im Ost- oder im Westlappischen ursprünglicher ist. 
im geringsten Stellung nehmen zu wollen, stelle ich nur fest, dass die Verschiedenheit der Erscheinung deutlich darauf hinweist, dass nach der Entstehung des Stufenwechsels der Osten und der Westen je in eigener Richtung verschiedene Wege gegangen sind.

Auf eine bekannte Lauterscheinung möchte ich noch die Aufmerksamkeit lenken. Die alten Verbindungen ${ }^{*} n t,{ }^{*} m p$ und $*^{*} \eta k$ kommen als gesetzmässig crhalten nur im Kolalappischen vor, z. B. $\iota, \bar{n}^{t E}(\operatorname{lp} \mathrm{N}$ lod de) 'Vogel' usw.; siehe T. I. ITkoNEx MSFOu 39 S. 73 ff. Hier und da in verschiedenen Gegenden der lappischen Iialekte, in denen sonst regelmässig Assimilation stattgefunden hat, gibt es Nasalbildungen, die sehr alt sein können, obgleich die Wörter in der Hauptsache entlehnt oder deskriptiv sind. Es kann sein, dass die Assimilation in den zentralen lappischen Dialekten ihren Anfang genommen und von dort sich sowohl nach Osten wie nach Westen verbreitet hat, wie Bergsland gemeint hat; siche Grammatikk S. 56. Da die Assimilationsvertretung auch im Südlappischen als normal und durchgehend anzusehen ist, muss man jedenfalls konstatieren, dass die Kolalappischen Dialekte dem westlichen Einfluss schon damals entzogen waren, als das Südlappische noch fest zur nördlichen Einflusssphäre gehörte. Das Verschmelzen der Nasale mit homorganen Klusilen und die so entstandene Verkürzung des konsonantischen Elements an der Grenze zwischen der zweiten und dritten Silbe ist eine deutlich jüngere Erscheinung als der Stufenwechsel. In einem solchen lappischen Wort wie lpN suoladit 'stehlen' kann man die schwache Stufe nicht anders erklären als durch die Annahme, dass die zweite Silbe geschlossen war. In der Zeit der Entstehung des Stufenwechsels hat an der Grenze zwischen der zweiten und dritten Silbe entweder nd oder $d d$ gestanden, aber nicht ein einzelnes $d$. Dennoch gibt es im Südlappischen ein ebensolches einzelnes $d$ wie im Finnmarklappischen, z.B. Härjedalen $\operatorname{să}_{a}^{a} l a d{ }^{j} t$. Einen entsprechenden Fall sehen wir im Komparativ, wo $m b>b, \mathrm{z}$.B. $\operatorname{lpN}$ buorĕb, $\operatorname{lpS}$ buaŕab, $ə, \operatorname{lpK}$ puoreamp. Im Lichte derartiger Beispiele ist es schwer, nicht den Schluss zu ziehen, dass damals, als die Assimilation und die Konsonantenverkürzung durchgeführt wurden, alle westlichen Dia- 
lekte eine feste Ganzheit bildeten und dass nur die im äussersten Osten befindlichen Kolalappischen Dialekte (Akkala, Kildin, Ter) an der Entwicklung nicht teilnahmen. Das Inari- und Skoltlappische kennen die oben dargestellte Lautveränderung an der Grenze der zweiten und dritten Silbe, was auf ein hohes Alter der Erscheinung hindeutet.

Die von mir behandelten Erscheinungen zeigen nicht direkt das Vorhandensein eines urlappischen Stufenwechsels. Ihr Beweiswert ist dennoch, wie mir scheint, indirekt recht bedeutend. Da der Stufenwechsel der ostlappischen und der westlappischen Dialekte ganz klar gemeinsamer Herkunft ist, ist er zu einer Zeit entstanden, wo diese Dialekte einander sehr nahe standen. Aber da vollauf Grund besteht anzunehmen, dass die ostlappischen Dialekte und besonders ihre östlichsten Mundarten sich von der übrigen Gemeinschaft schon damals getrennt haben, als das Südlappische mit seinen nördlichen Nachbarn noch fest verbunden war, folgt daraus, dass es schwer, um nicht zu sagen, geradezu unmöglich ist zu verstehen, wie es vom Stufenwechsel hätte frei bleiben können.

Nunmehr ist es angebracht, über die Erscheinungen zu sprechen, die direkter auf den Stufenwechsel hinweisen. In der Entstehungszeit des Stufenwechsels sind die Lautverhältnisse in vieler Hinsicht andere gewesen als heut. Die schwache Stufe kam anfänglich, ebenso wie im Urfinnischen, nur vor einer ursprünglich geschlossenen Silbe vor, die starke stufe dagegen vor einer offenen Silbe. So verhalten sich die Dinge nirgendswo mehr. Infolge einiger Lautveränderungen sind viele ursprünglich geschlossene Silben offene geworden und ungekehrt, ohne dass das noch den Stufenwechsel beeinflusst hätte. Man kann sagen, dass der Stufenwechsel, nachdem es nun einmal so gekommen war, aufgehört hatte, ein lebendiges, rein phonetisches, an die ursprünglichen Voraussetzungen gebundenes Prinzip zu sein. Einige dieser Lautveränderungen sind so alt, dass das Südlappische an ihnen teilgenommen hat. So labe ich ja schon oben einen Fall wie suoladit erwähnt, der schwerlich auf die Weise zu erklären ist, dass wir behaupteten, das südlappische $d$ sei ganz und gar das Ergebnis einer selbständigen Entwicklung. 
Mit grutem (irumd muss man besonders auf den wichtigen Umstand hinweisen, den ERkKi ITKoves in Struktur und Entwicklung der ostlappischen Quantitätssysteme S. 10-11 behandelt. Nach seiner Auffassung, der man sich mit Recht anschliesst, gab es im Urlappischen an der Grenze der zweiten und dritten (resp. der vierten und fünften) Silbe keinen quantitativen Stufenwechsel. Den im Ostlappischen auftretenden qualitativen Wechsel bestimmter Konsonanten kann man seiner Meinung nach jedoch auf das Urlappische zurückführen. In der in Rede stehenden Stellung liegt immer die kurze Stufe vor, so dass eimen ursprünglichen einfachen Konsonanten ein einfacher Konsonant und eine Geminata ein halblanger Konsonant vertritt. S. 11 sagt er: "Übrigens erscheint im Lappischen vor dem auf der Grenze der zweiten und dritten Silbe stehenden Vertreter einer ursprünglichen Geminata im Stamm des Wortes die nichtwechselnde schwache Stufe, obwohl die zweite Silbe durch Kürzung der Geminata schon früh offen geworden ist. Dieser Umstand zeigt seinerseits, dass der Stufenwechsel schon damals nur als traditionelles Erbe einer früheren Sprachform fortlebte; die Faktoren, die seinerzeit den Stufenwechsel hervorriefen, hatten schon aufgehört, aktiv zu sein." An der Kürzung der auf den Vokal einer unbetonten Silbc folgenden Geminaten hat auch das aus $h t$ und $p t$ entstandene $t$ teilgenommen. Von allen diesen Lautveränderungen gibt es auch im südlappischen Spuren. Das Südlappische nimmt bekanntlich insofern eine besondere stellung ein, als dort die Konsonanten im Wortauslaut gut erhalten geblieben sind wie ${ }^{*} m,{ }_{n},{ }^{*} k$, die in den nördlicheren Dialekten lautgesetzlich nach einer unbetonten Silbe regelmässig geschwunden sind. Es ist sicher, dass der Schwund des Auslautskonsonanten in einigen Fällen urlappisch ist. Wie z.B. $\operatorname{lpN} g \bar{a} m \hat{a}$, GASg. gābmâyâ zeigt, ist der Stufenwechsel älter als der Schwund des Auslautskonsonanten. Die Vertretung im Südlappischen ist in einigen Fällen eine analoge Verallgemeinerung, wozu natürlich das Verschwinden des Stufenwechsels seinerseits Anlass gegeben hat. Sich ein Bild von den Einzelheiten in dieser Frage zu machen, ist jedoch natürlich sehr schwierig.

Ebenso wie die schwache Stufe vor einer offenen Silbe auf- 
tritt, findet sich die starke Stufe in den Dialekten mit Stufenwechsel in einigen Fällen auch dann, wenn sich im Späturlappischen schon eine sekundäre geschlossene Silbe gebildet hatte. Es dürfte kein Zweifel darüber bestehen, dass z.B. das Imperfekt Ip N bōttim, lpRöros $b_{\propto} \ddot{o t} t^{\prime}$ am schon im Späturlappischen diese Form gehabt hat, so dass das Vorkommen der starken Stufe in diesen Formen unerklärlich würde, wenn der Stufenwechsel noch ein lebendiges Prinzip gewesen wäre. Dieses Imperfekt sei nur ein Beispiel unter vielen ähnlichen. Wer behauptet, der Stufenwechsel sei eine jüngere Erscheinung als das Urlappische, nimmt eine schwere Last auf seine Schultern, denn alle Fälle, in denen die schwache und die starke Stufe im Widerspruch zu den ursprünglichen Verhältnissen auftreten, müssen natürlich erklärt werden.

Einige dialektgeographische Feststellungen, die mit dem Stufenwechsel verbunden sind, sprechen recht deutlich dafür, dass der Stufenwechsel tatsächlich auf dem Wege des Ausgleichs in dem Südlappischen geschwunden ist. Der Stufenwechsel tritt noch vollkommen ausgebildet im Pitelappischen auf, also verhältnismässig weit im Süden. Von diesem Dialekt nach Süden hin beginnen die Ausgleichserscheinungen sichtbar zu werden, und zwar in der Weise, dass der Ausgleich ein um so grösseres Gebiet erfasst, je weiter man nach Süden geht. In der Mundart von Semisjaur im Dialekt von Arjeplog, der zwischen dem Pite- und Umelappischen steht, ist der Stufenwechsel nach einem etymologisch kurzen Vokal der ersten Silbe in der $x x$-Reihe ausgeglichen, so dass die starke Stufe allgemeinherrschend geworden ist. Die Verhältnisse liegen also in grossen Zügen so, als wenn man im Finnischen deklinieren würde ukko: ukkon, aber taakka: taakan, joki: joen, palkka: palkan. Nach Lagercrantz kann man Stufenwechsellosigkeit zuweilen auch nach einem langen Vokal antreffen. Wenn wir aus dem Bereich dieses Dialekts weiter nach Süden auf das Gebiet der Mundart von Sorsele uns begeben, also eigentlich schon auf das des Umelappischen, können wir feststellen, dass auch die $x$-Reihe den Stufenwechsel nach einem kurzen Vokal verloren hat und dass die $x$-Reihe auch quantitativ der $x x^{-}$ Reihe gleich geworden ist. Dagegen trifft man den Wechsel 
nach langem Vokal in allen Reihen an. In Dialekt von Mala herrschen nach Schlachter in der Hauptsache dieselben Verhältnisse. Wenn die Stufenwechsellosigkeit des südlappischen die ursprünglichen Verhältnisse wiedergäbe, wäre es natürlich anzunehmen, dass die in den ihnen nahen nördlichen Dialekten feststellbaren recht begrenzten Stufenwechselfälle einen primitiven Wechsel verträten, der sich dann immer weiter ausgebreitet hätte. Ein solcher Gedanke ist jedoch ganz unmöglich, denn die Fälle von Stufenwechsel im Umelappischen sind eindentio Ergebnis des Ausgleichs, worauf auch der Umstand hindeutet, dass die $x$-Reihe nach kurzem Vokal sich wie die $x x$-Reihe zu verhalten begonnen hat. Bergsland gibt zu, dass die Vertretung in den Dialekten von Sorsele und Malå sich auf keine andere Weise erklären lässt als dadurch, dass man einen Ausgleich dess Stufenwechsels voraussetzt. Wenn man aber, je mehr man von Norden her nach Süden kommt, deutlich beobachtet, dass der Ausgleich immer weitere Ausmasse annimmt und einen immer grösseren Teil des Konsonantenbestandes umfasst, kommt man nicht an dem Gedanken vorbei, dass diese gleichmässig über das Gebiet hin sich verstärkende Ausgleichung im süılen schliesslich den ganzen Konsonantismus erfasst hat. Im Schwedischlappischen ist der Stufenwechsel in der Hauptsache quantitativ und nur zum geringen Teil qualitativ. Dass der quantitative Ausgleich zunächst nach kurzem Vokal stattfand, wo die Konsonantenlänge zur festen Regel wurde, weist deutlich auf skandinavischen Einfluss hin. Die skandinavischen Sprachen kennen ja nicht kurze Konsonanten nach kurzem Vokal. Die $x y$-Reihe konnte natürlich ihr Wechselverhältnis nach kurzem Vokal zäh beibehalten, denn hierbei war kein Widerspruch zu den Quantitätsprinzipien der fremden Sprache vorhanden.

Für den Ausgleich des Stufenwechsels, der natürlich nicht nur als Folge lautgesetzlicher Entwicklung, sondern in grossem Masse auch durch analoge Verallgemeinerung zustande kam, spricht sehr stark die Tatsache, dass man im Südlappischen ganz regelmässig analoge Verallgemeinerungen vorfindet, namentlich dann, wenn der Nominativ und die obliquen Kasus infolge einiger früher Lautveränderungen sich weit voneinander 
entfernt haben. Beispiele finden sich reichlich in allen Dialekten. Es seien hier nur einige erwähnt zum Zeichen dafür, wie gesetzmässig solche Verallgemeinerungen sind; siehe BJöRN Collisder Lappisches Wörterverzeichnis aus Härjedalen.

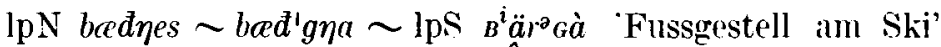
$\operatorname{lpN} \operatorname{dot} t \hat{a} \sim \operatorname{dok}^{1} t \hat{a} g \hat{a} \sim \operatorname{lp} \mathbf{S}$ dak'tô $g_{u} \varepsilon$ 'Taucher'

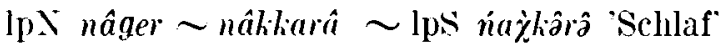

IpN sâco $\sim$ sâtunâ $\sim \operatorname{lps}$ suuuâne 'Erweiterung eines Flusses"

$$
\begin{aligned}
& \operatorname{lpX} \text { suolâ } \sim \text { suollâyâ } \sim \operatorname{lps} \text { sucọ̆tâ }{ }_{u} \varepsilon \text { 'Dieb' } \\
& \text { lpN suolo } \sim \text { sul lu } \sim \text { lps's sỏte 'Insel' }
\end{aligned}
$$

Fälle wie lpN rappen IpS re ${ }^{\varepsilon}$ hpane 'Rauchöffnung im Zelt', IpN šimer IpS ša p̀mârà 'Mlesserrücken' zeigen, dass der Endrokal des Nominativs analog ist. Der Schwund des Endvokals in diesen Fällen ist eine sehr alte Erscheinung, wie die Schwachstufigkeit des Stammkonsonantismus erweist. Mit derartigen den Stamm betreffenden Verallgemeinerungen verbindet sich, wit schon unsere Beispicle zeigen, ohne weiteres auch die Stufenverallgemeinerungr. Wenn wir also beim Südlappischen von Verallgemeinerung sprechen, bewegen wir uns nicht in einem bloss theoretischen Bereich. Überzeugende Beispiele zeigen, dass die Verallgemeinerung tatsächlich eine gewöhnliche Erscheinung ist. Welcher Umstand eine solche Verallgemeinerung, die am häufigsten dazu fülurt, dass der Stamm der obliquen Kasus Nominativ wird, verursacht hat, ist schwer genau zu sagen. Man muss sich jedoch daran erinnern, dass das Südlappische derjenige von den lappischen Dialekten ist, in den fremder Einfluss am tiefsten hat eindringen können. Die Lappen sprechen in der heutigen Zeit hauptsächlich Schwedisch und Norwegisch und haben es so schon recht lange gemacht. Die Zahl derer, die ihre ursprüngliche Muttersprache recht mangelhaft beherrschen, ist ausserordentlich gross. Im sprachgebrauch der Einzelnen kommt eine auffallende Unsicherheit zum Vorschein, und alle Forscher von I. HALÁsz angefangen, mussten feststellen, dass in den Dialekten nicht die innere Festigkeit vorhanden ist, die doch sonst eine verhältnismässig normale Erscheinung ist. Der Umstand, dass der fremde Ein- 
fluss viel Yeues mit sich gebracht und alte Systeme zerstört hat, bedeutet keineswegs, dass hier an der Peripherie trotz aller verhältnismässig jungen Veränderungen nicht auch vieles vom alten Erbe sich erhalten konnte. Das Südlappische ist in dieser Beziehung nicht einzigr in seiner Art.

Wenn man der urlappischen Herkunft des Stufenwechsels auf den Grund gehen will, muss man besonders beachten, dass im Südlappischen der sogen. suffixale stufenwechsel noch deutlich zu beobachten ist, z.B. ist die Endung des Partitirs nach einer betonten Silbe -htie, abor nach dem Vokal einer unbetonten Silbe $-d a,-\dot{r} a$, z.B. dähtie $\sim$ nuppada, nuppara. Val. aluch die 1. Pl. Präs. liepie $\sim$ boåtaba. Der radikale und der suffixale Wechsel sind dermassen Parallelerscheinungen, dass sie deutlich zusammengehören. Dirse Feststellung hat in Bezug auf dic ostseefinnischen Sprachen Iaunr Postr gemacht; siehe Grundzüge der livischen Lantgeschichte S. 231, Anm. 1. Wenn wir die Auffassung haben, dass der rildikile und der suffixale Wechsel zusammengehören, bedeutet dies, dass sie im Grunde unter dem Einfluss derselben Ursachen entstanden sind. Wenn wir an der 'Theorie festhalten, dass dir schwache Stufe nach einer ungeraden Silbe von der Betonung der folgenden Silbe herrührt,

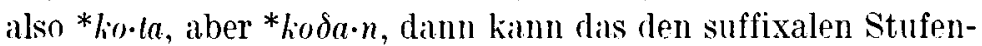
wechsel nicht erklären, denn z.B. in dem Fall *kalada ist die schwache Stufe auf keinen Fall eine Folge davon, dass die dritte Silbe betont gewesen wär. Wenn wir dagegen roralsssetzen, dass die schwache Stufe sich dann bilden konnte, wenu vor dem Konsonanten keine starke Betonung lag. brauchen wir offensichtlich keinen grundsätzlichen Enterschied zwischen dem radikalen und suffixalen Wechsel anzunehmen. Im Fall *kota war die erste Silbe stark betont und der Vokal endete mit stark geschnittenem Akzent, weshalb die Voraussetzungen für das Erhalten des Klusils günstig waren. Im Fall *kodan dagegen hat die zweite Silbe schon wegen der grösseren Quantität einen 'Teil der starken Betonung der ersten Silbe für sich verlangt. Mit *kota verglichen war *kodan auf beiden Silben relativ gleichmässig betont und gleichzeitig war der Vokal der ersten Silbe am Ende abgeschwächt, was alles Voraussetzung für die Erscheinung der Abschwächung war. Die Vorausset- 
zungen für die Abschwächung sind ganz offensichtlich in solrhen unbetonten Stellungen wie in *kalada. Vgl. SeтÄL ̈̈ JSFO॥ 14, 3 S. 22 ff., Brengsuad Stud. Sept. 2. \$. 4, Lauri Posti FIF 31 s. 76 ff.

Wegen all dieser Gesichtspunkte konnte ich zu keinem andtren Ergebnis kommen als zu dem, dass alle lappischen Dialekte auf eine Sprachform zurückgehen, wo der Stufenwechsel sich sowohl in der radikalen wie in der suffixalen Erscheinungsweise herausgobildet hatte. Das Verschwinden des Stufenwechsels ist keineswegs bloss durch analoge Verallgemeinerungr zustandegekommen, sondern direlite Iantveränderungen haben ihrerseits auch in dieser Richtung gewirkt. Einstweilen können wir jedoch nicht im einzelnen sagen, was eine Folge der lautgesetzlichen Entwicklung und was eine der Analogie ist. Eine Frage grundsätzlicher Art möchte ich jedoch berïhren. In den Diskussionen über den Stufenwechsel ist es üblich geworden. als Arqumente gegen analoge Verallgemeinerung Fäll» heranzuziehen, wo in der Flexion nur e i n e Stufe vorkam. Wenn z.B. in irgendeinem Wort nur die schwache Stufe mörrlich gewesen ist und wenn nichts anch nur annähernd vou starker Stufe zu finden war, hielt man die Verallgemeinerung der starken Stufe: für ganz unmöglich. So könnte es scheinen, aber hier kann man die Bemerkung nicht unterlassen, dass eine solche Argumentation .Junggranmatikertum in Reinkultur ist. Es ist klar, dass Stufenverallgemeinerungen, die in Wörtern stattfanden, in denen beide Stufen vorkamen, und die in jedem Fall in der Mehrzahl gewesen sind, unmittelbar auf das System selbst rewirkt haben. Die Verallgemeinerungen haben auch die Fälle miterfasst, in denen kein Stufenwechsel vorgekommen war. I)a die Analogie die Vertretung der starken Stufe auch in die schwache Stufe hineingebracht hat, ist es klar, dass Altes und Neues nebeneinander bestanden hat, vgl. z.B. die in Dialekt von Jukkasjärvi nebeneinander vorkommenden Formen odas und opas, die sogar bei denselben Personen aufgezeichnet werden konnten. Wenn die schwache stufe endgültig beiseite gedrängt ist und z.B. an die Stelle eines Spiranten oder stimmhaften Klusils regelmässig cin stimmloser Klusil tritt, konnten die wenigen Fillo mit der schwachen Stufe, noben denen in 
keiner nahestehenden Form eine starkstufige Klusilvertretung gefunden wird, sich nicht erhalten, sondern haben gleichzeitig damit die schwache Stufe verloren. Wenn der Stufenwechsel nur ein Wechsel von Varianten (Allophonen) ist, so ist der Ausgleich sehr leicht zu verstehen, vgl. S. 287.

Der lappische Stufenwechsel ist trotz einiger besonderer Züge im Grunde der gleiche wie der der ostseefinnischen Sprachen. Die schwache und die starke Stufe werden durch die gleichen Voraussetzungen bestimmt. Es ist schwer, sich vorzustellen, dass der Stufenwechsel in jeder von beiden ganz sclbständig entstanden sei. Man muss dagegen mit gutem Grund die Anfänge schon in der finnisch-lappischen Ursprache suchen, wie wenig oder wie sehr zusammenhängend eine solche Ursprache auch immer gewesen sein mag.

Mir scheint es auch weiterhin am treffendsten anzunehmen, dass besonders die Geminataklusile den ersten Ausgangspunkt für den Wechsel gebildet haben, wie ich im Virittäjä 1951 dargelegt habe. LAUR Posti hat freilich die Bemerkung vorgebracht, dass eine anfängliche Abschwächung der Geminataklusile, auch wenn eine solche stattgefunden hätte, noch nicht bedeutet hätte, dass die Geminataklusile den einfachen Klusilen so nahe gekommen wären, dass ihre Abschwächung unvermeidlich gewesen wäre, damit beide Reihen voneinander getrennt geblieben wären. Er weist darauf hin, dass im Späturfinnischen ein klarer Unterschied zwischen der schwachen Stufe der $x x$-Reihe und der starken der $x$-Reihe bestanden hat, und dass dieser Unterschied in einigen ostseefinnischen Sprachen noch vorhanden ist. So etwas habe ich natürlich nicht gemeint. Die Reihen bleiben voneinander scharf getrennt, solange die schwachen Stufen beider voneinander getrennt bleiben; denn erst die Gleichheit der in der gleichen Stellung vorkommenden Laute macht die analoge Vermengung möglich. In der Stufenwechseltheorie wurde oft recht willkürlich von einem Reihenübergang gesprochen, ohne dass man dem Umstand Beachtung schenkte, dass die schwache Stufe der $x x$ Reihe und die starke der $x$-Reihe im Sprachsystem funktionell cine andere Stellung einnehmen. Die lautliche Gleichheit ist etwas anderes als die Gleichheit der Funktion. Es handelt sich 
darum, dass im Lautsystem einer Sprache eine bestimmte Beziehung zwischen der $x x$-Reihe und der $x$-Reihe herrscht. Wenn in der einen Reihe eine Abschwächung oder Verstärkung crfolgt, ist oft die entsprechende Erscheinung in der anderen Reihe festzustellen. In vielen finnisch-ugrischen Sprachen sind alle ursprünglichen Geminataklusile einfache Klusile geworden, entsprechend sind die einfachen Klusile abgeschwächt und stimmhafte Laute geworden. Wichtig war, dass beim Stufenwechsel das Verhältnis zwischen den Reihen erhalten blieb. Wenn also in der $x x$-Reihe eine Abschwächung in der schwachen Stufe stattfand $(x x>\bar{x} x)$, hat das keinen Einfluss auf die starke Stufe der $x$-Reihe, sondern nur auf deren schwache (also $x>\breve{x}$ ).

Wenn man die Bezichungen zwischen dem Urfinnischen und dem Urlappischen vergleicht, stellt man leicht fest, dass eine vollkommene Entsprechung nicht vorhanden ist. Man darf jedoch nicht vergessen, dass die Forschung hinsichtlich des Späturfimmischen in ihren Rekonstruktionen weiter gelangt ist als in denen über das Urlappische. Es ist noch viel Einzelarbeit zu leisten, bis wir sagen können, welche urlappischen Ausgangsformen anzunehmen sind. Hinter diesen muss dann das gesucht werden, was der urfimnischen und urlappischen Sprachform gemeinsam ist. Im folgenden greife ich einige Erscheinungen auf, die mit diesen Fragen zusammenhängen.

\section{Die $x$-Reihe}

Die $x$-Reihe vertritt nach der herrschenden und gut begründeten Meinung ursprüngliche einfache Konsonanten. In den lappischen Dialekten tritt in der starken Stufe allgemein eine Geminata auf, während ein einfacher Konsonant in der schwachen Stufe erscheint (z.B. lpN guolle $\sim$ guole, vgl. fi. kala $\sim$ lalan). Im südlappischen herrscht nach etymologisch kurzem Vokal dieselbe Vertrrtung wie bei der ursprünglichen Geminata, nach langem Vokal wiederum steht ein einfacher Konsonant, z.B. $i u h k_{u} \varepsilon$ 'Fluss', aber $i \tilde{o} k i i^{h}$ 'teilen'. Wiksund erklärte die südlappische Vertretung einfach damit, dass die starke Stufe nach einem kurzen Vokal, die schwache aber nach 
einem langen Vokal allgemein geworden sei. Eine derartige Erklärung dürfte kaum richtig sein, sondern es handelt sich natürlich um klare Lautveränderungen. Nach langem Vokal hat eine quantitative Verkürzung des Stammkonsonanten stattgefunden und entsprechend ist der Konsonant nach kurzem Vokal verlängert worden. Neben diesen lautlichen Veränderungen sind dann analoge Ausgleichungen eingetreten, die, wie wir oben konstatierten, keineswegs nur die Paradigmen betrafen, in denen die starke Stufe mit der schwachen wechselt. Was den späturlappischen Wechsel angeht, erscheint es irgendwie als sicherer anzunehmen, dass in der schwachen Stufe ein kurzer einfacher Konsonant gestanden hat und in der starken Stufe eine deutlich davon verschiedene Quantität des Konsonanten. Ich nehme an, dass die Rekonstruktion $\dot{x} \sim x$ ungefähr das Richtige trifft.

Was die Klusile betrifft, so setzt man bei diesen neben dem quantitativen Wechsel einen klaren qualitativen Wechsel voraus, und dieser Zug rührt offensichtlich aus der finn-lappischen Ursprache her. Der Wechsel könnte im Urlappischen der folgende gewesen sein: ${ }^{*} \dot{k} \sim{ }^{*} g,{ }^{*} \grave{i} \sim{ }^{*} d,{ }^{*} \grave{p} \sim{ }^{*} b$. Die anderen Konsonanten haben keinen qualitativen Wechsel gekannt, so dass auch das ursprüngliche spirantenpaar $\grave{\delta} \sim \delta$ einem rein quantitativen Wechsel unterlag. Den Spiranten als Vertreter der schwachen Stufe der Klusile hinzustellen, ist sehr schwer. Die zentralen Dialekte des Finnmarklappischen ebenso wie das Ostlappische kann man zwar auf einen solchen Ausgangspunkt zurückführen, wenigstens ohne allzu gewaltsame Erklärungen. Im Terlappischen wären dann die Spiranten zu Verschlusslauten geworden, z.B. GASg. joga von jogk ${ }^{A}$ 'Fluss' = Kld. $j \bar{o} \gamma a$, Sk. $j \bar{o} \gamma \gamma^{\hat{v}}$; siehe ERKkI ITKonen FUF 27 S. 153. Aber die westlichen Dialekte weichen beträchtlich ron diesem Bild ab. Und so hat man begründeten Zweifel gegen diese Rekonstruktion vorbringen können. In den westlichen Dialekten ist der Klusil in der schwachen Stufe recht gewöhnlich. Man kann sagen, dass die Grenze der klusilen und spirantischen Vertretung in den zentralen finnmarklappischen Dialekten zwischen den Mundarten von Kautokeino und Karasjok verläuft. In der Mundart von Karasjok tritt der Spirant oder 
jedenfalls die darauf zurückzuführende Vertretung ebenso auf wie in der Mundart von Polmak, aber in der von Kautokeino steht der Spirant nur in der schwachen Stufe von * $i$. Dagegen erscheint als schwache Stufe von $* \dot{k}$ und $*_{\dot{p}}$ ein halbstimmhafter Mediaklusil, z.B. Kt (Nielsen) juhlià $\grave{a}^{h} \sim$ Präs. 1. P. Sg. juā̄n 'drink', Gohpà ${ }^{h} t \sim$ Gosān 'hollow out', aber' joht' $\imath^{h} t \sim$ jodan 'be in motion'. In den schwedischlappischen Dialekten steht regelmässig in der schwachen Stufe auch der Wörter mit ${ }^{*} \dot{t}$ ein Klısil, ein mehr oder weniger stimmhafter Mediaklusil oder ein klarer Tenuisklusil, also $d, \quad b$ oder $t$. Dieses Klusilgebiet reicht vom Süden bis zum Dialekt von Jukkasjärvi-Rautasvuoma, wo ein Spirant oder eine am ehesten auf ihn zurückzuführende Vertretung neben der klusilen Vertretung vorzufinden ist. Sogar bei demselben Sprecher kann $D$ mit $\delta$ wechseln. Der Schwund, der in diesem Dialekt anzutreffen ist, wie auch das vibrationslose $x$, hat sich aus dem $\delta$ entwickelt; siehe Näheres bei Collurver The lappish dialect of Jukkasjärvi S. 133. Der Dialekt von Jukkasjärvi schliesst sich nach Collinder fester an die finnmarklappische als an die lulelappische Gruppe an, obgleich auch in der letzteren gemeinsame Züge reichlich zu konstatieren sind; siche a.a.O. $273 \mathrm{ff}$. WIKLUND hat in seiner bekannten Stufenwechseluntersuchung (siehe MO 7) festgestellt, dass die Wörter mit ursprünglichem $* i$ und $* \delta$, die sich in den Dialekten von Ter und Kildin infolge Analogie der schwachen Stufe gänzlich miteinander vermischt haben, anderswo voneinander getrennt geblieben sind. Das ist der Fall besonders in den schwedischlappischen Dialekten und auch in denen, die keinen Stufenwechsel haben. So lautet das ein ursprüngliches $* i$ aufweisende $\mathrm{lpN}$ boattet $\sim$ badam 'kommen' im Lulelappischen pāhtèt $\sim p \bar{a} t \bar{a} v$, aber das ein ursprüngliches $* \grave{\delta}$ aufweisende $1 \mathrm{pN}$ oad̋et $\sim$ oadam 'schlafen' lautet $\bar{a} t \bar{t} t$ $\sim \bar{a} t \bar{a} v$, so dass der quantitative Stufenwechsel verschwunden ist. Die weiter im Süden herrschenden Verhältnisse sind noch klarer, da auch die schwache Stufe gesondert verharrt, z.B. lpP bōhtet bōdau, ōddet ōdau, lpRöros boåtajh boåtam, oårojh oåram. In Wörtern mit ursprünglichem $* \delta$ troffen wir also eine Vertretung an, die nicht nur von den Wörtern mit *i abweicht, sondern auch leicht auf einen Dentalspiranten zurückführbar ist. Im 
Pitelappischen hat der Spirant sich erhalten, weiter im Süden ist er zu $r$ geworden, in Härjedalen steht jedoch an Stelle des $r$ ein $d$. Im Lulelappischen entspricht dem Dentalspiranten $t$, wie gesagt, sowohl in schwacher wie in starker Stufe, aber es verdient Beachtung, dass in der Überdehnungstufe $d d$ steht. Nach Wiklund kann gar nicht die Rede davon sein, dass in Wörtern mit ursprünglichem $*_{i}$ im Urlappischen in der schwachen Stufe * $\delta$ gestanden hätte. Dagegen muss man von einem stimmhaften Klusil ausgehen und dann entsprechend in Wörtern mit $* k$ und $* \dot{p}$ auch von stimmhaften $g$ und $b$. Die Forscher haben sich im allgemeinen mit den Gedanken Wiklunds nicht

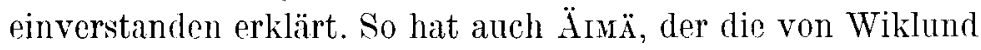
vorgebrachten Argumente anfangs für bindend lielt und deshalb glaubte, in der Vertretung des Dialekts von Sompio etwas gefunden zu haben, was die Vermutung des schwachstufigen Klusils gestützt hätte, siehe JSFOu 30, 30a s. 53 Anm., später entschieden gegen diese Theorie polemisiert. In Virittäjä 1922 S. 9 kommt Äimä zu dem Ergebnis, dass der urlappische Spirant sich zu einer Media entwickelt und seine Stimmhaftigkeit im Prinzip ebenso wie der Spirant nach einem stimmhaften Konsonanten verloren habe. Das ist nach ihm allgemein in den Dialekten geschehen, in denen man auch sonst eine Reduktion der Stimmhaftigkeit in der schwachen Stufe findet. Die gebietsmässige Parallelität ist zwar nicht vollständig, aber es ist denkbar, dass die lautliche Entwicklung in irgendeinem Zentrum angefangen hat und dass sie anfangs nur einige Fälle umfasst hat. Die netue Vertretung hätte sich dann vom Zentrum aus in immer grösser werdenden Kreisen verbreitet. Später hätte dann ein Ausgleich nach verschiedenen Richtungen hin stattgefunden. Äimä ist gezwungen anzunehmen, dass die Reduktion der Stimmhaftigkeit auch die Wörter mit ${ }^{*} \delta$ betroffen habe, und nach ihm ist ja der durch die Lautveränderung in der schwachen Stufe entstandene Wechsel im Dialekt Jukkasjärvi-Rautasvuoma am besten erhalten geblieben, wo opas mit der Form oddasak der starken Stufe wechselt. Die Vertretung der schwachen Stufe sei in den einen Dialekten, wie besonders im Lulelappischen, in der starken Stufe verallgemeinert worden, in anderen dagegen habe die Vertretung dor star- 
ken Stufe die Vertretung der gesetzmässigen sclıwachen Stufe verdrängt, besonders südlich von dem Dialekt von Arjeplog. Es ist, wie mir scheint, recht schwer, den Erwägungen Äimäs zuzustimmen. Das Wesentlichste ist jedenfalls, wenn wir von dem spiranten ansgehen, sein Wandel in einen Klusil. Dass dann stellenweise der aus dem Spirant hervorgegangene Klusil seine Stimmhaftigheit verloren hat, ist eine andere Sache, und sie ist offensichtlich späteren Ursprungs, da das ja nicht überall, wo der Spirant zum Klusil wurde, stattgefunden hat. Man muss wohl einer Meinung mit Wiklund und Bergsland darüber sein, dass die Wörter mit $* \dot{t}$ in der Schlüsselstellung stehen.

Ich meinerseits habe mich auch auf den standpunkt derer grestellt, die den Spiranten als die Ausgangsform der schwachen Stufe bezeichnet haben. Ich habe gedacht, dass der in den westlichen Dialekten in Erscheinung tretende Wandel in Klusilo auf skandinavischen Einfluss zurückgehen könne und dass der Wechsel $t t: d$ in der Gegend von Kautokeino so zu erklären soi, dass $d d$ in der starken Stufe den Wandel in einen Klusil nicht mitgemacht und durch Analogie auch auf die schwache stufe gewirkt habe, die ihrerseits auf die schwache Stufe der Wörter mit 1 Einfluss gehabt habr. Aber nachdem ich die Frage immer und immer wieder durchdacht und mich grïndlich mit der Kritik, die Wiklund und Bergsland an der Spirantentheorie geübt haben, vertraut gemacht hatte, habe ich mich den Rekonstruktionen Wiklunds angeschlossen. Es ist ganz offensichtlich, dass die Wörter mit $t$ und $d$ nicht so genau voneinander getrennt geblieben wären, wie sie sind, wenn die schwachen Stufen identisch gewesen wären. Im Kolalappischen stellen wir fest, dass überall, wo in den Wörtern mit $t$ in der schwachen Stufe ein Spirant auftritt, die Wörter gänzlich zusammengefallen sind und eine Vermengung allgemein zu beobachten ist. Auch im Lulelappischen kommen Übergänge vor, z.B. nà̀ $a$ 'Stiel', vgl. lpN nâddâ; siehe Collixder L IWDG S. 135 ff. Abcr in den Dialekten, bei denen kein begründeter Anlass besteht zu vermuten, dass die schwachen Stufen der Wörter mit $t$ und $d$ ursprünglich identisch gewesen sind, ist auch keine Vermengung festzustellen, worauf Bergsland eigens aufmerksam macht, vgl. a.a.0. 36. Da meiner Auffassung nach die 
Wechselverhältnisse der Klusile nach Liquida oder ILalbvokal den Wechselverhältnissen der Klusile in intervokalischer Stellung entsprochen haben und da es keineswegs wahrscheinlich ist, dass auch in diesen in der schwachen Stufe ein Spirant vorgekommen sei, sondern ein mehr oder weniger stimmhafter Klusil, ist auch dieser Lmstand geeignet, uns in der Annahme zu bestärken, dass der Spirant als die schwachstufige Entsprechung des Klusils auf dem Gebiet der lappischen Sprache in allen Stellungen eine verhältnismässig junge Erscheinung ist. Jan kann schwerlich annelımen, dass z.B. ${ }^{*} \beta$, die schwachstufige Entsprechung des $p$, Iange Zeitläufte hindurch sich von $v$ hätte genau getrennt halten können. Im Lappischen von Kildin und Ter existiert es noch getrennt, aber im Kolta- und Inarilappischen wie auch in den östlichen Dialekten von Finnmarken ist es wie im Finnischen mit dem i verschmolzen. In den westlichen lappischen Dialekten ist die schwache Stufe des $p$ gewöhnlich ein Klusil. Ich sehe von keiner Seite her eine wesentliche Schwierigkeit, wenn wir mit Wiklund als Ausgangsformen der schwachen Stufe die stimmhaften $b, d, g$ annehmen. Es bedarf keiner erklärenden Worte, dass es unmöglich ist, über die Stufe der Stimmhaftigkeit etwas zu sagen. Es kann doch sein, dass man ebensogut vou einem halbstimmhaften Mediaklusil ansgehen könnte. So ist es meiner Meinung nach in jedem Fall natürlich zuerst gewesen, wahrscheinlich war der urfinnisch-urlappische Wechsel eben dieser Art.

In den Diskussionen über die Spirantentheorie hat eine gewisse Rolle das merkwürdige südlappische jiŕrado 'Morgen' gespielt, das vermutlich, wie vorgebracht worden ist, die $n t-$ Ableitung des Verbs jijtic- 'erscheinen, aufgehen (Sonne)' ist; siehe Collinder LWDG S. 142. Hier scheint $\dot{r} \dot{r}$ tatsächlich einem früheren Dentalspiranten zu vertreten. Bergsland, der die Etymologie gutheisst, meint, dass die Form möglicherweise eine Entlehnung aus dem Norden ist. Erkki Itkonen begrïndet seine Auffassung folgendermassen: "Die Erhaltung des $\delta(>r)$ in dieser Ableitung rührt natürlich davon her, dass jirrado im Sprachbewusstsein schon früh von seinem Stammwort isoliert worden ist; das $t$ des letzteren hat folglich keine Voraussetzungen gehaht, sich an Stelle von $* \delta$ zu verall- 
gemeinern. Obschon jirradz nach seinem Lautbestand den Erwartungen nicht ganz entspricht (im Inneren des Wortes ist $r$ statt $j$ ), bestätigt dies noch nicht die Vermutung yon Berogsland, dass das Wort aus den nördlicheren Dialekten als Entlehnung ins Südlappische gekommen sein, FUF 32 Anz. S. $57-$. In dem Worte gibt es jedoch noch anderes von der Regel Abweichendes als das ŕr an Stelle des $j r$. Im Dialekt von Härjedalen rertritt den früheren Dentalspiranten $d d$, aber wie Bergsland bemerkt, gibt es von dem Wort jiŕrada nur Formen mit $r$. Es versteht sich, dass man auf einen solchen Ausmahmefall nichts Entscheidendes aufbauen kann.

Bergsland heisst jedoch nicht einmal die Rekonstruktion Wiklunds gut, sondern nimmt an, dass sowohl in der starken wie in der schwachen stufe ein stimmloser Konsonant gestanden habe. Er begründet seinen Standpunkt in einer sehr interessanten Weise und auf Grund eines wichtigen Lautverhältnisses. Da ich seinem Beweisgang nicht habe zustimmen können, fühle ich mich veranlasst, auf die Frage einzugehen, zumal sie rom Standpunkt der ganzen finnisch-ugrischon Lautgeschichte her bedeutsam ist.

Schon lange ist das Interesse der Forscher durch den Umstand errogt worden, dass man im Lappischen in einigen Wörtern den Wechsel $k k: g$ als Entsprechung zum Schwund im Finnischen vorfindet. Die bekanntesten von diesen sind lpN jukliât: jugâm $\sim$ fi. juoda, lpN sulkiât : sugâm $\sim$ fi. soutaa. Spuren von einer ursprünglichen Konsonantenvertretung sind auch in anderen finn.-ugrischen sprachen vorhanden, wenn auch der rorzufindende Konsonant geschichtlich vieldeutig ist. So kann z.B. in mordw. vijems 'führen', das dem fi. viedä und lp Kld. $v i \bar{G} h p^{\delta}: r i \gamma a^{m}$ id. entspricht, das $j$ ebensogut ein ursprüngliches $j$ wie auch $k$ oder sogar $\eta$ oder $\gamma$ vertreten. SETäLä hat den Gedanken ausgesprochen, dass bei diesen Wörteru und ausserdem bei einigen anderen, bei denen es im Lappischen nicht die Vertretung $k k: g$ gibt, ron einem ursprünglichen ${ }^{*} \gamma$ auszugehen ist, das in der starken Stufe quantitativ länger und klanglich kräftiger gewesen sei als in der schwachen Stufe. Die Vertretung der schwachen Stufe führte sodann zum Schwund. Die Vertretung im Lappischen beruht natürlich auf einem Wech- 
sel der Reihe, der durch die Identität der schwachen Stufen in den $k$ - und $\gamma$-Wörtern vermittelt worden ist, siehe FUF 12 Anz. S. 41. Die Vermutung über das ursprüngliche $\gamma$ würde natürlich damit gut zusammenpassen, dass es auch die ursprünglichen Dentalspiranten $\delta$ und $\delta^{\prime}$ gab. Setälä sagt ja auch ausdrücklich, dass er zu diesem Ergebnis ungefähr zur gleichen Zeit gekommen ist, als er das Vorkommen von Dentalspiranten, dic mit Klusilen nicht wechseln, bewiesen hatte. PAasones hielt es auch für möglich, dass in diesen Fällen schon in der uralischen Ursprache in der starken Stufe ein stimmhafter Palatal aufgetreten sei; siehe Beitr. S. 59-60. Wenn diese Theorie zutrifft, wäre das ein ausserordentlich starker Beweis dafür, dass der phoncmatische Stufenwechsel im Urlappischen vorhanden war, denn das Vorkommen des Palatalklusils in der starken Stufe ist offenbar mit dieser Theorie gar nicht anders zu erklären. Leider kann man es keineswegs für klar halten, dass es in diesen Wörtern niemals eine andere Vertretung als die im Lappischen gezeigte gegeben habe. Bergsland hat in seiner erwähnten Untersuchung die beachtenswerte Behauptung aufgestellt, dass auch im Lappischen die in Rede stehenden Wörter in der Zeit des Urlappischen von den $k$ Wörtern getrennt waren, und dass diese Getrenntheit im Südlappischen nachzuweisen ist. Wenn die Erscheinungen wirklich im Sinne Bergslands zu deuten wären, erhielte die Theorie Setäläs von der Ursprünglichkeit des $\gamma$ eine ausgezeichnete starke Stütze, um nicht zu sagen, geradezu eine Bestätigung. Es handelt sich eigentlich um zwei Wörter, nämlich jukkât 'trinken' und sukkât 'rudern'. Dagegen findet man im südlappischen duokza- 'verkaufen', das Bergsland richtig mit fi. tuoda, mordw. tuje-, tuvo-, ostj. tù-, wog. tü-li verbindet, dasselbe $k$ vor, das dem ursprünglichen $k$-Laut entspricht, z.B. $i_{a} \not{o} k i^{h}=\mathrm{fi}$. jakaa usw. Dass in den südlappischen Entsprechungen von jukkât und sukkât eine andere Vertretung vorhanden ist als in duokz-, hängt mit der Geschichte des Vokals der ersten Silbe zusammen. Im Südlappischen kommen bekanntlich in einigen Fällen $u w$ und $i j$ als Entsprechung des $u$ und $i$ des Finnmarklappischen vor. Es handelt sich offenbar um eine spät entstandene Diphthongierung. Die zur $x$-Reihe 
des Urlappischen gehörenden $t$ und $p$ haben durch diese Lautveränderung eine Vertretung erhalten, der eigentlich die Geminata des Finnischen entspricht. So lautet denn auch IpN ittet : idam im Südlappischen jijtie- und $\operatorname{lpN}$ suppe : suve (= fi. haapa) süupie. Von dieser Vertretung weichen heut die Entsprechungen von IpN jukkât und sukkât ab. Sie sind in die Medienreihe übergegangen und lauten näml. juwg ${ }^{2-}$, suwg ${ }_{c}{ }^{--}$. Hasselbrink, der in seiner Dissertation als erster auf diese aussergewöhnliche Vertretung aufmerksam geworden ist, erklärt die Erscheinung, die er in der Mundart von Vilhelmina festgestellt hat, so, dass es sich um eine Verallgemeinerung der schwachen Stufe handle. Nach Bergslands Ansicht ist es sehr befremdend, wenn eine Verallgemeinerung der schwachen Stufe gerade bei diesen beiden Wörtern vorausgesetzt wird, die nach Setäläs Theorie eben die ursprünglichen Spirantenfälle vertreten. Besser würde man sie dadurch erklären, dass man voraussetzt, sie seien auch ursprünglich eine Besonderheit gewesen. Bergslands Gedanken hat sich Erkki Itkones angeschlossen. Nach seinen Überlegungen ist der ursprüngliche Unterschied zwischen den $k$ - und $\gamma$-Wörtern überhaupt im Lappischen ausgeglichen worden, wie Setälä annahm, zugunsten von $k$. Dagegen sei die fürs Südlappische typische Diphthongierung älter als der Zusammenfall von $k$ und $\gamma$, und auf $\gamma$ zurückgehendes $g$ habe dadurch, dass es zur zweiten Komponente der Konsonantenverbindung wurde, sekundär seine Stimmhaftigkeit bewahrt; siehe FUF 30 s. 5.

Unleugbar scheint das auf den ersten Blick sehr überzeugend, aber in Wirklichkeit ist das Bild nicht ganz so einfach. Zunächst muss man sagen, dass im Südlappischen, wie schon viele Forscher bemerkt haben, der Unterschied zwischen $w^{1} \%$ und $w^{\prime} g$ usw. oft recht klein ist. Die Fälle sind schwer voneinander zu trennen, da der stimmlose Vokal (die Präaspiration) oft fortbleibt und der Mediaklusil stimmlos geworden ist. Ein bestimmter Unterschied der Reihen besteht dennoch häufig. So ist im Dialekt von Röros offensichtlich der Unterschied zwischen Fortis und Lenis phonematisch; siehe Bergsland, Grammatikk S. 49. Dasselbe ist in der Mundart von Vilhelmina gewöhnlich der Fall. Aber es wäre ganz falsch zu behaup- 
ten, dass betreffs jukkât und sukkât im Südlappischen regelmässig und allgemein eine andere Vertretung vorkomme als z.B. in den Fällen suppe und ittet. Es ist zunächst klar, dass in der Mundart von Vilhelmina die Sache sich so verhält. Die Aufzeichnungen von Hasselbrink, Wiklund und Lagercrantz sind ganz übereinstimmend. Aber z.B. im Dialekt von Röros verhält es sich nicht so. Bergslands wichtigste Sprachmeisterin, Julie Axmann, die nach ihm ausserdem die einzige ist, die den Dialekt rein spricht, siehe Røros-samiske tekster S. 165, gebraucht juw'ke- und suw'ke-. Der Dialekt von Röros ist ganz der gleiche wie der von Härjedalen. Bei Collinder gibt es keinen Unterschied zwischen süpè und $j \bar{u} k_{u} i^{j}$ in dieser Hinsicht. Die Mundart von Vilhelmina gehört zu den nördlichsten in den süddialekten und ihr Nachbardialekt im Norden gehört schon zum Umelappischen. In der Mundart von Sorsele, wo das urlappische $u$ gleichfalls diphthongiert ist, finden wir

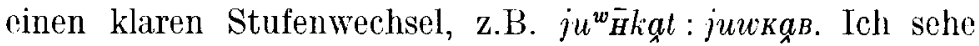
nichts Gewaltsames in der Vermutung Hasselbrinks, dass in der Mundart von Vilhelmina tatsächlich eine Stufenverallgemeinerung stattgefunden habe. Die uns zugänglichen Aufzeichnungen zeigen mehr als klar, wie gefährlich es wäre, auf Grund der Fälle sukkât und jukkât weitgehende Schlussfolgerungen zu ziehen. Auch in den ursprünglichen $w k$ und $i k$ Fällen können dialektal recht schwankende Vertretungen vorkommen. Z.B. zu lpN čuoi'kâ 'Mücke' lauten die südlappischen

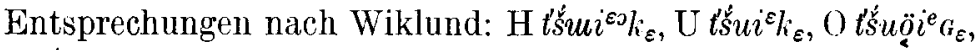
F t'śü̊isk. Als Entsprechung zu lpN čuoi'gât 'auf Schneeschuhen laufen' erwähnt Halász aus dem Dialekt von Undersåker u.a. čuóikih, čuóihki usw. Wenn man das ganze südlappische Gebiet ins Auge fasst, kann kein grundsätzlicher Unterschied einerseits zwischen den Fällen jukkât, sukkât und andrerseits den mit ittet, suppe sein.

Das Lappische kann also der Annahme, dass in den Fällen julikat usw. sowohl in der starken wie in der schwachen Stufe ein Spirant gestanden habe, keinerlei Berechtigung geben. Das Wahrscheinlichste ist, dass in den behandelten Fällen ursprünglich ein $k$ vorhanden gewesen ist, das im Urlappischen mit $g$ gewechselt hat. ERKKI ITKones ist sicherlich im Recht, wenn 
er annimmt, dass das $k$ im Urfinnischen nach langem Vokal in den $e$-stämmigen Wörtern und vor allem in deren konsonantstämmigen Formen vokalisiert worden und geschwunden sei. Wie all dies in Einzelheiten vor sich gegangen ist und über welche Zwischenstufen der Schwund erreicht worden ist, das ist hingegen nicht klar, obgleich andrerseits irgendeine Spirantenstufe wahrscheinlich scheint.

\section{Die Wechselverhältnisse der $x x$-Reihe}

Die Lappologen sind bisher im grossen ganzen einer Meinung darüber gewesen, dass die schwache Stufe der $x x$-Reihe im Späturlappischen mit der starken Stufe der $x$-Reihe identisch gewesen ist. Die Bezeichnung war nicht einheitlich; die einen bezeichneten den Konsonanten als lang, die anderen als halblang, aber eine Greminata mit kurzem Anfang wurde nicht angewendet. Der Wechsel wäre demnach gewesen $\grave{x} x: \bar{x}$ oder $\grave{x} x: \grave{x}$. Natürlich hat man nicht angenommen, dass es von Anfang an so gewesen sei. Man war ja seitens des Ostseefinnischen daran gewohnt, bei der schwachen Stufe der $x x$-Reihe von einer Geminata mit kurzem Anfang auszugehen, während man für die starke Stufe der $x$-Reihe einen einfachen Konsonanten verzeichnete. Bergsland hat in den herrschenden Frieden Störungen gebracht, und es ist ihm anscheinend geglückt, hier leichter seine Kollegen zu überzeugen. Die Frage ist jedoch sicherlich noch nicht von der Tagesordnung abgesetzt. Was nun die Dialekte angeht, in denen der Stufenwechsel noch vorkommt, lassen sie sich mühelos auf die von der alten Theorie vorausgesetzten Ausgangsformen zurückführen.

Bergsland richtet seine Aufmerksamkeit darauf, dass im Südlappischen die $x x$ - und die $x$-Reihe nach langem Vokal streng voneinander getrennt blieben. Es gibt eine grosse Menge Wörter, namentlich wörter der $x$-Reihe, bei denen in allen Flexionsformen nur die starke Stufe vorkam, z.B. davara, IpN davver (= finn. tavara), noaimala lpN njoammel 'Hase', vič̌əra $\mathrm{lpN}$ vaččer (=finn. vasara) usw. Diese Argumenticrung richtet sich in erster Linie gegen die Annahme Wiklunds, dass der Stufenwechsel im Südlappischen dadurch ausgeglichen 
worden sei, dass die starke Stufe nach kurzem Vokal, die schwache Stufe aber nach langem Vokal verallgemeinert worden sei. Aber wenn ich auch zugebe, dass es nicht möglich ist, den z.B. in rioamala auftretenden einfachen Konsonanten für eine Verallgemeinerung der schwachen Stufe zu halten, kann ich doch nicht einsehen, dass dies zwingend zeigt, dass die schwache Stufe der $x x$-Reihe und die starke Stufe der $x$-Reihe phonetisch nicht identisch gewesen seien.

Zu allererst muss man sich an die Verhältnisse im Umelappischen, dem nächsten Nachbar des Südlappischen erinnern, denn in diesem Dialekt ist der Stufenwechsel nach kurzem Vokal ganz und gar ausgeglichen. Man hat Grund anzunehmen, dass das auf dem Wege zum Verschwinden des Stufenwechsels befindliche Umelappische gerade die Stufe vertritt, die am ehesten dem heutigen Stand des Südlappischen voraufgegangen ist. Ich stelle mir vor, dass die Entwicklung vor allem so vor sich ging, dass die langen Konsonanten in der schwachen Stufe: der $x x$-Reihe und in der starken Stufe der $x$-Reihe lautgesetzlich gelängt wurden. Eine Folge davon war, dass der Stufenwechsel

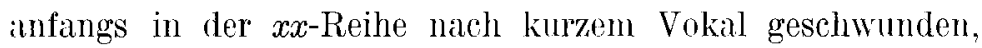
aber in der $x$-Reihe (quantitativ) weiterhin in Kraft geblieben ist. Dass im Umelappischen anch in der $x$-Reihe kein Wechsel vorkommt wie in der Mundart von Semisjaur, beruht meiner Meinung nach klar auf analogem Ausgleich; der Stufenwechsel ist nach kurzem Vokal ausgeblieben und die Lautgestalt des Konsonantismus hat sich in der Weise eingebürgert, dass nach kurzem Vokal nur ein langer Konsonant stehen konnte. Die Analogie ist natürlich nicht eine ganz unbedingte Voraussetzung, denn es ist ja denkbar, dass die Kraft, die die quantitative Längung der Konsonanten verursachte, fortgesetzt ihren Einfluss ausübte. Ein solches interessantes Wort wic Malå sihpèhka 'Ski' (vgl. IpN sâbek, fi. sivalka) ist keineswegs unbedingt nur als Ergebnis einer lautgesetzlichen quantitativen Veränderung zu erklären. Da im Dialekt von Malå nach langem Vokal der Vertreter der schwachen Stufe der $p$-Wörter (also ${ }^{*} b$ ) ein Mediaklusil ist, würde man als Ergebnis der Entwicklung wohl einen langen Mediaklusil erwarten. Die Analogie ist in diesem Fall meiner Meinung nach durchaus möglich, eben wegen 
der ciründe, anf die ich oben hinwies. Als deg Stufenwerhel besonders unter dem Einfluss der $x$-Reihe auch in der $x$-Reihe nach kurzem Vokal sich auszugleichen begann, erschienen in der schwachen Stufe der $x$-Reihe neben $G, B, D$ immer häufiger $h l, h p, h t$ vor allem in den Wörtern, die daneben eine starke Stufe hatten, aber dann natürlich auch in den selteneren, bei denen es diese nicht gab. Der Systemzwang führte dann zur Verallgemeinerung. $O b$ die Entwicklung sich einzig und allein auf lautgesetzlichem Wege oder, wie ich es für wahrscheinlicher halte, sowohl durch Lautgesetze wie durch Analogie vollzog, jedenfalls ist im Malålappischen die schwache Stufe der $x x$-Reihe nach langem Vokal phonetisch mit der starken stufe der $x$-Reihe identisch, z.B. àhki : $\bar{a}^{\prime} G a ̀ u$ 'Grossmutter',

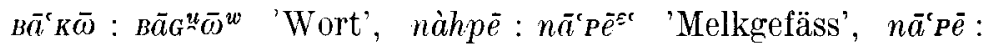
$n \bar{a} B \dot{e}^{u}$ 'Nabel'. Ich kann gar nicht begreifen, warum das Südlappische nicht ebensogut auf diese Wechselbeziehung zurückgehen könnte. Ich halte das ausserdem für sehr wahrscheinlich, da, wie ich sagte, alle lappischen Dialekte sich mühelos auf diesen Lautstand zurückführen lassen.

Es besteht vielleicht Anlass, noch einmal auf die prinzipielle Frage hinzuweisen, ron der schon die Rede war. Der Umstand, dass die $x x$ - und die $x$-Reihe von einander klar getrennt geblieben sind, obgleich die schwache Stufe der ersteren und die starke Stufe der letzteren gleich waren, ist recht gut verständlich. Als rein phonetische Phänomene sind sie einander gleich, aber ihre Funktion ist eine ganz verschiedene. Die schwache Stufe der $\boldsymbol{x} x$-Reihe kommt in bestimmten Flexionsformen vor, sagen wir z.B. oft im Genetiv-Akkusativ. In einer solchen Stellung kann die starke Stufe der $x$-Reihe nicht auftreten; sie kommt in Fällen vor, in denen die folgende Silbe ursprünglich offen war. Trotz der Gleichartigkeit als Phänomene bleiben sie streng von einander getrennt. Deshalb sind die Möglichkeiten analoger Verallgemeinerungen auch ganz verschiedene. Wenn die schwache Stufe der $x x$-Reihe analoge Verallgemeinerung erfährt, betrifft das natürlich nicht die starke Stufe der $x$-Reihe. Ausserdem gibt es auch dafür Beispiele, dass die reine lautliche Entwicklung in verschiedene Richtungen gehen kann. In einigen finnmarklappischen Dialekten hat sich die starke Stufe 
von goppe: gobe zur gleichen Form entwickelt wie die starke Stufe von gop'pe:goppe, während die schwache Stufe der $x x^{-}$ Reihe verschieden blieb, was, wie mir scheint, ein guter Beweis dafür ist, wie die phonetisch gleichen heteronymen Glieder der Reihen sich in verschiedene Richtungen entwickeln konnten. Gestützt auf all das, was ich oben gesagt habe, möchte ich deshalb weiterhin an der alten Rekonstruktion $\grave{x} x: \grave{x}$ und $\grave{x}: x$ festhalten. Das betrifft also nur das Späturlappische.

\section{Fragen der xy-Reihe}

Die Verhältnisse in Lappischen weichen, namentlich was die Vertretung der postkonsonantischen Klusile angeht, in interessanter Weise von den Verhältnissen im Finnischen $a b$. Nach stimmhaftem Konsonanten treffen wir im Finnischen in der Hauptsache den gleichen Wechsel an wie zwischen Vokalen. Nach Vokalen flektiert man ukko : ukon und zum Beispiel nach einer Liquida pallka : palkan. Hier hat, wie man vermutet, in beiden Fällen die (reminata der starken Stufe mit einer schwachstufigen (ieminata mit kurzem Anfangsteil gewechselt, also *ukko: *ukkkon und *palkka: *palkkian. Im Urlappischen kam nach einer Liquida überhaupt keine Geminata vor, sondern statt deren ein langer einfacher Klusil sowohl in der starken wie in der schwachen Stufe. Nach CoLlinder lautete del Wechsel im Urlappischen $* i \grave{k} \sim * i \grave{k}$.

Betreffs der einfachen Klusile muss man im Finnischen auch den gleichen Wechsel nach einem stimmhaften Konsonanten roraussetzen wie nach Vokalen, und deshalb rekonstruiert man z.B. ${ }^{*}$ joki : ${ }^{*}$ joyen und ${ }^{*}$ jalka $:{ }^{*}$ jalyan. Im Lappischen hat es hier einen stimmlosen Klusil anscheinend gar nicht gegeben, sondern offensichtlich einen nicht wechselnden halbstimmhaften Mediaklusil. Nach Collinders Rekonstruktion war das

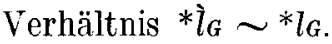

Der Wechsel ist also rein quantitativ gewesen und hat nur die erste Komponente der Konsonantenverbindung betroffen. Der Mediaklusil ist in beiden Fällen kurz. Nach einigen Forschern hat es im Urlappischen in der schwachen Stufe einen Spiranten gegeben, siehe z.B. Äмӥ in Virittäjä 1922 S. 9. 
Erkki Itronen sagt FUF 26 Anz. S. 57, dass er der Rekonstruktion Collinders zustimme und den im Ostlappischen feststcllbaren Wechsel $l_{G} \sim l \gamma$ für wahrscheinlich später halte als das Urlappische, vgl. auch FUF 27 S. 144, Anm. 1. Er nimmt später in einem anderen Zusammenhang, siehe FUF 30 S. 8 an, dass es im Frühurlappischen nach einem Halbvokal (und vermutlich auch nach einer Liquida) den Wechsel ${ }^{*} \dot{w} G:{ }^{*} u \gamma$ gab. Solche in ostlappischen Dialekten rorkommende Fälle

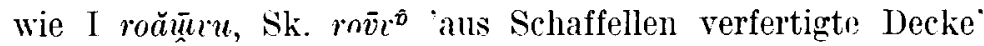

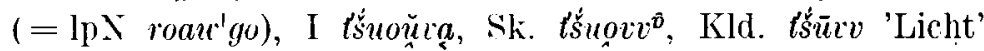
$(=\operatorname{lpN}$ čuow gâ), wo also eine Vertretung vorhanden zu sein scheint, die sowohl in der starken wie in der schwachen Stufe einen Palatalspiranten voraussetzt, werden nach Itkonen am natürlichsten eben dadurch erklärt, dass man die Verallgemeinerung der schwachen stufe voraussetzt; siehe Äмӓ JSFOU 23, 25 S. 13 ff., FUF 6 S. 181 ff., T. I. Itkonen VKA S. 45, 60, Wikluni) FUF 6 S. 5 ff., Bergsland Stud. Sept. 2 S. $43 \mathrm{ff}$.

Die Verhältnisse im Frühurlappischen liegen natürlich im I) unkeln, aber dic Verschiedenheiten, die in den Verhältnissen des Fimnischen und Iappischen festzustellen sind, kann man meiner Meinung nach doch ziemlich ungezwungen erklären. Für die Entwicklungr des lappischen Konsonantismus ist eine offensichtliche Verstärkung der Quantität in der starken Stufe charakteristisch. Es bestelit, wie mir scheint, auch nicht der geringste Grund dafür, von irgendwelchen uralten finnischugrischen halblangen Konsonanten auszugehen (also nicht von *kala : *kalan, sondern von einem kurzen nicht wechselnden $l$ ). Die Verhältnisse im Iappischen werden von denen im Finnischen gerade durch diese Verstärkung der Quantität in der starken Stufe, die den ganzen Konsonantenbestand erfasst hat, also auch die Geminata und die Konsonantenverbindungen, unterschieden. Gerade dies ist, soweit ich sehe, der wesentliche Unterschied zwischen den Systemen des Urlappischen und Urfinnischen. Und aus diesem wesentlichen Unterschied lassen sich, wie mir scheint, auch viele andere im Stufenwechsel auftretende Verschiedenheiten erklären. In der Sprachform des 
Frühurlappischen stand also meiner Auffassung nach in der starken Stufe $x, x x, x y$ und $x y y$. Ich vermute nämlich, dass auch das Lappische auf eine solche Ausgangsform zurückgeht, in der nach einer Liquida und nach einem Halbvokal ein Geminataklusil aufgetreten ist. Solche Ausgangsformen setzt meiner Meinung nach ganz klar auch das Mordwinische voraus (vgl. tarka $=$ fi. tarkia, jalgo = fi. jalka usw.). So sind also im Frühurlappischen, wenigstens dann, wenn Klusile die zweite Komponente waren, die $x y$ - und die $x y y$-Reihen ganz wie im Urfinnischen. Als dann im Urlappischen in der starken Stufe die Verstärkung der Quantität bogamn, wurde aus $x$ zunächst $\dot{x}$. Aus $x x$ entwickelte sich die lange Geminata $\grave{x} x$. In der $x y y$-Reihe hingegen konzentrierte sich die Verstärkung der Quantität, wie mir scheint, auf die erste Komponente, aber diese konnte nur auf Kosten der zweiten Komponente morklich stärker werden. Die so eingetretene Kürzung hat anscheinend schliesslich dazu geführt, dass der Klusil nach der crsten Komponente sowohl in der starken wie in der schwachen Stufe gleichlautend wurde, und so ist bei diesen der Wechsel geschwunden. Nur die Quantität der ersten Komponente hat die starke Stufe von der schwachen unterschieden. Vermutlich lag die Silbengrenze in der $x y y$-Gruppe zwischen $x y$ und $y$, also $x y_{\wedge} y$. Nach der Quantitätsentwicklung hat dann die erste Komponente $x$ allein über den zur ersten Silbe gehörenden 'T'eil dominiert und so könnte lautgesetzlich die $x y y$-Reihe geschwunden und an ihre Stelle die $\grave{x} y$-Reihe getreten sein. In der ursprünglichen $x y$-Reihe (also z.B. jalka) hat die Verstärkung der ersten Komponente in der starken Stufe möglicherweise eine qualitative Abschwächung der zweiten Komponente verursacht. Jedenfalls ist es ganz offensichtlich, dass die Reihen der Geminata und der einfachen Klusile in deutlicher Beziehung zueinander stchen, vgl. meinen Artikel in Virittäjä 1951. Wenn ein Geminataklusil abgeschwächt wird, ist anzunehmen, dass in der entsprechenden Umgebung auch eine Abschwächung

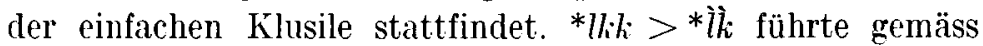
dem Systemzwang anscheinend dazu, dass $* l_{k}$ zu $* \grave{l}_{G}$ wurde.

Wenigstens auf westlappischem Gebiet kinn man gut von 
der von Collinder voriulsgesetzten Ausgangsform $* \grave{l}_{G} \sim{ }^{*} l_{G}$ aulsgrehen. Der in vielen I)ialekten vorkommende Wechsel del Stimmhaftigkeit zwischen der starken und schwachen Stufe ebenso wie die in verschiedener Richtung verlaufende Quantitätsentwicklung lassen sich als späte Erscheinungen erklären.

Wenn also im Frühurlappischen nach stimmhaftem Konsonanten dieselben Verhältnisse geherrscht haben wie nach Vokal, wäre die Form des Stufenwechsels demnach gewesen: ${ }^{*} l k \sim$ ${ }^{*} l y,{ }^{*} l l \sim{ }^{*} l d,{ }^{*} l p \sim{ }^{*} l b$ usw. Wenn die Längung des crsten 'Teils der Konsonantenverbindung die Schwächung des Geminataklusils in der Verbindung ${ }^{*} l k \cdot k(>* i k)$ bewirkte, dann fand dieselbe Abschwächung auch in der Verbindung $* l k\left(>{ }^{*} \grave{l}_{G}\right)$ statt. Das war geeignet. dip Klusile der starken und der schwalchen Stufe einander nahezubringen, da ausserdem der Stufenwechsel hauptsächlich auf dio erste Komponente sich verlagrert hatte. Die Voraussetzungen für einen fortgesetzten Ausgleich waren grareben, besonders da anch in der ursprünglichen Geminatenreihe ein solcher Ausgleich stattfand.

Das oben Vorgebrachte zusammenfassend halte ich es also für offensichtlich. dass dor Stufenwechsel als rein phonetischr Erscheinung im Lappischen und im Finnischen gemeinsamen Trsprungs ist. In der schwalchen stufe fand eine quantitativ" Abschwächung dor freminataklusile statt. wogegen die einfachen Klusile sich in der Richtung der Stimmhaftigkeit entwickelten, was an einigrn stellen später die Abschwächung des Klusils zum Spiranten zur Folge hatte, ja sogar zu seinem Schwund geführt hat. Anfangs gah es im finnisch-lappischen Stufenwechsel nichts anderes (remeinsames als das Schwächerwerden der Klusile vor geschlossener Silbe und an der Grenze: der zweiten Silbe wie auch in einigen Fällen ein Schwächerwerden in derselben Richtung an der Grenze der zweiten und dritten Silbe. Die besonderen Züge des lappischen Stufenwechsels haben sich durch die allgemeine quantitative Verstärkung der starken stufe herausgebildet. Das hat einen alle Konsonanten erfassenden Wechsel verursacht und dann auch entsprechend einige Phänomene in der Entwicklung der Klusilc hervorgebracht, 
Das folgende Schema reranschaulicht meine Auffassung:

Die fimnisch-lappische Ursprache

$$
\begin{aligned}
k k & \sim \check{k} k \\
k & \sim \check{k} \\
l k k & \sim l \grave{k} k \\
l k & \sim l \check{k}
\end{aligned}
$$

Das Urlappische $\dot{\jmath} \dot{k} \sim \grave{k}$

$\grave{k} \sim g$

$\grave{l} \dot{k} \sim i k$

$\grave{l}_{G} \sim l g$

Paavo Ravila 